\title{
MORE PRESSURE IN THE FINITE ELEMENT DISCRETIZATION OF THE STOKES PROBLEM
}

\author{
Christine Bernardi ${ }^{1}$ And Frédéric Hecht ${ }^{1}$
}

\begin{abstract}
For the Stokes problem in a two- or three-dimensional bounded domain, we propose a new mixed finite element discretization which relies on a nonconforming approximation of the velocity and a more accurate approximation of the pressure. We prove that the velocity and pressure discrete spaces are compatible, in the sense that they satisfy an inf-sup condition of Babuška and Brezzi type, and we derive some error estimates.
\end{abstract}

Résumé. Pour le problème de Stokes dans un ouvert borné bi- ou tridimensionnel, on propose une discrétisation par un nouvel élément fini mixte, qui utilise une approximation non conforme de la vitesse et une approximation plus riche de la pression. On prouve que les espaces discrets de vitesse et de pression sont compatibles, au sens qu'ils vérifient une condition inf-sup de Babuška et Brezzi, et on en déduit des majorations d'erreur.

Mathematics Subject Classification. 65N30, 76D07.

Received: October 22, 1999.

\section{INTRODUCTION}

The Navier-Stokes equations modelizes the flow of a viscous incompressible fluid, and much work has been done concerning their finite element discretizations. However the numerical analysis of such methods relies on the study of the corresponding discretizations of the linear Stokes problem which is considered in this paper. Since its formulation in the primitive unknowns of velocity and pressure results into a saddle-point problem, the well-posedness of both this system and any Galerkin type discretization of it is derived from an inf-sup condition of Babuška [2] and Brezzi [5] type, which ensures the compatibility of the spaces of velocities and pressures. This condition is well-known for the continuous problem, see for instance [11, Chap. I, §2], however proving it for the discrete problem is the key point for checking its stability.

In the finite element framework, the inf-sup condition has been proven for a large number of elements including the Crouzeix-Raviart element [8], the mini-element and the Taylor-Hood element. We refer to [11, Chap. II] for an extensive review of these elements and the corresponding inf-sup conditions. The idea of the proof is similar in most cases: starting from fixed discrete spaces of velocities and pressures which have the desired approximation properties, some further functions are added to the space of velocities in order to ensure that there is no spurious mode for the pressure. An inf-sup condition linking the resulting space with the space of pressures is then established. Most often the constant which appears in the inf-sup condition is checked to be

Keywords and phrases. Finite elements, Stokes problem, inf-sup condition, divergence-free basis functions.

1 Analyse Numérique, C.N.R.S. \& Université Pierre et Marie Curie, B.C. 187, 4 place Jussieu, 75252 Paris Cedex 05, France. e-mail: bernardi@ann.jussieu.fr

(c) EDP Sciences, SMAI 2000 
independent of the discretization parameter. We also quote two arguments which are of great help for the proof of this inf-sup condition: the Boland and Nicolaides idea [4] consists in proving this condition separately on the spaces of restrictions of discrete velocities and pressures to local subdomains and on reduced spaces of velocities and pressures on the whole domain; Verfürth's idea [14] allows for deriving the inf-sup condition from a first one with wrong norms, thanks to local approximation properties. Both of them can be used, for instance, for the inf-sup condition related to the Taylor-Hood element.

We start from a rather different point of view. We fix the discrete space of velocities to be the same as for the Crouzeix-Raviart element and recall that the corresponding discretization is nonconforming, in the sense that this discrete space is not contained in the variational one. Next, we assume that, in opposite to discrete velocities, the discrete pressures are continuous and we intend to work with the largest possible space of such pressures. The term "largest possible" must be understood in the sense that all the degrees of freedom of the discrete velocities are needed to prove the inf-sup condition. As explained in [9] and [7] in the slightly different framework of finite volumes, replacing the space of piecewise constant pressures of the Crouzeix-Raviart element by this larger and smoother discrete space is essential when working with geophysical flows, where the Coriolis acceleration must be taken into account. The use of smooth pressures is then necessary to respect the geostrophic equilibrium. It is also necessary for optimizing the geometry of the fluid domain when the optimization criterion involves local gradients of the pressure.

This paper is mainly devoted to the proof of the inf-sup condition for this new element. In a first step, we introduce a semi-orthogonal decomposition of both the spaces of velocities and pressures and investigate the inf-sup condition for each discrete subspace of pressures. One of the proofs relies on Verfürth's argument. In a second step, we derive the final inf-sup condition by using a modal analogue of the Boland-Nicolaides proof. This condition is not optimal, in the sense that it involves a constant which is not independent of the discretization parameter, however we think that the result we obtain is the best possible one and we give heuristic reasons for that. We deduce some convergence results and error estimates for the discrete velocity. In a final step, we propose an algorithm for solving the discrete Stokes problem, relying on the space of discrete velocities which are divergence-free in the finite element sense, and we characterize this space in the two-dimensional case.

An outline of the paper is as follows:

- In Section 2, we recall the variational formulation of the Stokes problem and we present the discrete problem relying on the new finite element.

- In Section 3, we prove some key results on the discrete spaces and present their decomposition as a direct sum of two subspaces.

- Sections 4 and 5 are devoted to the proof of inf-sup conditions related to the two subspaces of pressures.

- In Section 6, we derive the global inf-sup condition for the Stokes problem from the results of the preceeding sections, and we deduce the corresponding error estimates.

- In Section 7, we exhibit spaces of quasi-divergence-free discrete velocities in the two-dimensional case and describe the resulting algorithm for solving the discrete Stokes problem.

\section{The Stokes COntinuous AND Discrete Problems}

Let $\Omega$ be a connected and bounded open set in $\mathbb{R}^{d}, d=2$ or 3 , with a Lipschitz-continuous boundary. We consider the Stokes problem:

$$
\begin{cases}-\Delta \boldsymbol{u}+\operatorname{grad} p=\boldsymbol{f} & \text { in } \Omega, \\ \operatorname{div} \boldsymbol{u}=0 & \text { in } \Omega, \\ \boldsymbol{u}=\mathbf{0} & \text { on } \partial \Omega,\end{cases}
$$

where the unknowns are the velocity $\boldsymbol{u}$ and the pressure $p$. The data are a density of body forces $\boldsymbol{f}$ and, only for simplicity, we take homogeneous boundary conditions on the velocity. 
It is well-known that Problem (2.1) admits the following equivalent variational formulation: find $(\boldsymbol{u}, p)$ in $H_{0}^{1}(\Omega)^{d} \times L_{0}^{2}(\Omega)$ such that

$$
\begin{aligned}
\forall \boldsymbol{v} \in H_{0}^{1}(\Omega)^{d}, & a(\boldsymbol{u}, \boldsymbol{v})+b(\boldsymbol{v}, p)=\langle\boldsymbol{f}, \boldsymbol{v}\rangle, \\
\forall q \in L_{0}^{2}(\Omega), & b(\boldsymbol{u}, q)=0,
\end{aligned}
$$

where the space $L_{0}^{2}(\Omega)$ is defined by

$$
L_{0}^{2}(\Omega)=\left\{q \in L^{2}(\Omega) ; \int_{\Omega} q(\boldsymbol{x}) \mathrm{d} \boldsymbol{x}=0\right\},
$$

and $\langle\cdot, \cdot\rangle$ denotes the duality pairing between $H^{-1}(\Omega)^{d}$ and $H_{0}^{1}(\Omega)^{d}$. The bilinear forms $a(\cdot, \cdot)$ and $b(\cdot, \cdot)$ are defined on $H^{1}(\Omega)^{d} \times H^{1}(\Omega)^{d}$ and $H^{1}(\Omega)^{d} \times L^{2}(\Omega)$ by

$$
a(\boldsymbol{u}, \boldsymbol{v})=\int_{\Omega} \operatorname{grad} \boldsymbol{u} \cdot \operatorname{grad} \boldsymbol{v} \mathrm{d} \boldsymbol{x}, \quad b(\boldsymbol{v}, q)=-\int_{\Omega} q(\operatorname{div} \boldsymbol{v}) \mathrm{d} \boldsymbol{x} .
$$

Their continuity on these last spaces is obvious.

The ellipticity of the form $a(\cdot, \cdot)$ results from the Poincaré-Friedrichs inequality. And the following inf-sup condition on the form $b(\cdot, \cdot$,$) is standard [11, Chap. I, Cor. 2.4]: there exists a constant \beta>0$, only depending on the geometry of $\Omega$, such that

$$
\forall q \in L_{0}^{2}(\Omega), \quad \sup _{\boldsymbol{v} \in H_{0}^{1}(\Omega)^{d}} \frac{b(\boldsymbol{v}, q)}{|\boldsymbol{v}|_{H^{1}(\Omega)^{d}}} \geq \beta\|q\|_{L^{2}(\Omega)} .
$$

As a consequence, for any data $\boldsymbol{f}$ in $H^{-1}(\Omega)^{d}$, Problem $(2.1)$ has a unique solution $(\boldsymbol{u}, p)$ in $H_{0}^{1}(\Omega)^{d} \times L_{0}^{2}(\Omega)$.

Remark. It is readily checked that

$$
\forall \boldsymbol{v} \in H_{0}^{1}(\Omega)^{d}, \forall q \in L^{2}(\Omega), \quad b(\boldsymbol{v}, q)=\langle\operatorname{grad} q, \boldsymbol{v}\rangle,
$$

and the duality pairing in the previous line can be replaced by an integral whenever $\boldsymbol{v}$ and $q$ are smooth enough (for instance, when $\operatorname{grad} q$ belongs to $L^{2}(\Omega)^{d}$ ).

In order to write the discrete problem, we now assume that $\Omega$ is a polygon or a polyhedron and we introduce a regular family $\left(\mathcal{T}_{h}\right)_{h}$ of triangulations of $\Omega$ by (closed) triangles or tetrahedra, in the usual sense that:

- for each $h$, the intersection of two different elements of $\mathcal{T}_{h}$, if not empty, is a vertex, a whole edge or a whole face of both of them;

- the ratio of the diameter $h_{K}$ of an element $K$ in $\mathcal{T}_{h}$ to the diameter of its inscribed circle or sphere is bounded by a constant $\sigma$ independent of $h$.

As standard, $h$ denotes the maximal diameter of elements $K$ in $\mathcal{T}_{h}$.

We now fix two finite-dimensional subspaces $X_{h}$ and $M_{h}^{0}$ of functions in $L^{2}(\Omega)^{d}$ and $H^{1}(\Omega)$, respectively, which are polynomial on each element $K$ of $\mathcal{T}_{h}$. Our discrete problem relies on the variational formulation (2.2), it reads: find $\left(\boldsymbol{u}_{h}, p_{h}\right)$ in $X_{h} \times M_{h}^{0}$ such that

$$
\begin{array}{ll}
\forall \boldsymbol{v}_{h} \in X_{h}, & a_{h}\left(\boldsymbol{u}_{h}, \boldsymbol{v}_{h}\right)+b_{h}\left(\boldsymbol{v}_{h}, p_{h}\right)=\int_{\Omega} \boldsymbol{f}(\boldsymbol{x}) \cdot \boldsymbol{v}_{h}(\boldsymbol{x}) \mathrm{d} \boldsymbol{x}, \\
\forall q_{h} \in M_{h}^{0}, & b_{h}\left(\boldsymbol{u}_{h}, q_{h}\right)=0,
\end{array}
$$

where $\boldsymbol{f}$ is now taken in $L^{2}(\Omega)^{d}$ while the forms $a_{h}(\cdot, \cdot)$ and $b_{h}(\cdot, \cdot)$ are defined by

$$
a_{h}\left(\boldsymbol{u}_{h}, \boldsymbol{v}_{h}\right)=\sum_{K \in \mathcal{T}_{h}} \int_{K} \operatorname{grad} \boldsymbol{u}_{h} \cdot \operatorname{grad} \boldsymbol{v}_{h} \mathrm{~d} \boldsymbol{x}, \quad b_{h}\left(\boldsymbol{v}_{h}, q_{h}\right)=\sum_{K \in \mathcal{T}_{h}} \int_{K} \boldsymbol{v}_{h} \cdot \operatorname{grad} q_{h} \mathrm{~d} \boldsymbol{x} .
$$


Note that the difference between $a(\cdot, \cdot)$ and $a_{h}(\cdot, \cdot)$ only comes from the replacement of the integral on $\Omega$ by a sum of integrals on the $K$ in $\mathcal{T}_{h}$, while the modification of $b(\cdot, \cdot)$ involves a further integration by parts.

We need some notation to describe the discrete space $X_{h}$. Let $\mathcal{E}_{h}$ stand for the set of all edges in dimension $d=2$, faces in dimension $d=3$, of the elements of $\mathcal{T}_{h}$. We denote by $\boldsymbol{m}_{e}$ the midpoint, respectively the barycenter, of each $e$ in $\mathcal{E}_{h}$. The space $X_{h}$ is then the space of functions $\boldsymbol{v}_{h}$ in $L^{2}(\Omega)^{d}$ such that:

- their restriction to each $K$ in $\mathcal{T}_{h}$ belongs to $\mathcal{P}_{1}(K)^{d}$, where $\mathcal{P}_{1}(K)$ denotes the space of affine functions on $K$;

- they are continuous at each point $\boldsymbol{m}_{e}, e \in \mathcal{E}_{h} \cap \Omega$;

- they vanish at each point $\boldsymbol{m}_{e}, e \in \mathcal{E}_{h} \cap \partial \Omega$.

As already hinted, the corresponding finite element is exactly the one introduced in [8, Ex. 4].

However, the space $M_{h}^{0}$ is rather different from that in [8]. We make it precise later on and just indicate that it is a subspace with codimension 2 of the space

$$
M_{h}=\left\{q_{h} \in H^{1}(\Omega) ; \forall K \in \mathcal{T}_{h}, q_{h \mid K} \in \mathcal{P}(K)\right\},
$$

where $\mathcal{P}(K)$ stands for the space of dimension $d+2$ spanned by the polynomials in $\mathcal{P}_{1}(K)$ and the bubble function $\psi_{K}$ defined as the product of the barycentric coordinates on $K$.

Since the space $X_{h}$ is not contained in $H^{1}(\Omega)^{d}$, from now on, we work the mesh-dependent seminorm on $X_{h}$ :

$$
\left\|\boldsymbol{v}_{h}\right\|_{H_{h}^{1}(\Omega)}=\left(\sum_{K \in \mathcal{T}_{h}}\left|\boldsymbol{v}_{h}\right|_{H^{1}(K)^{d}}^{2}\right)^{\frac{1}{2}} .
$$

We recall from $\left[8\right.$, Lem. 2] that it is a norm on $X_{h}$. Note also that

$$
\left\|\boldsymbol{v}_{h}\right\|_{H_{h}^{1}(\Omega)}=a_{h}\left(\boldsymbol{v}_{h}, \boldsymbol{v}_{h}\right)^{\frac{1}{2}}
$$

Let us introduce the discrete kernel

$$
V_{h}=\left\{\boldsymbol{v}_{h} \in X_{h} ; \forall q_{h} \in M_{h}^{0}, b_{h}\left(\boldsymbol{v}_{h}, q_{h}\right)=0\right\}
$$

Then, for any solution $\left(\boldsymbol{u}_{h}, p_{h}\right)$ in $X_{h} \times M_{h}^{0}$ of problem (2.6), it is readily checked that $\boldsymbol{u}_{h}$ is a solution in $V_{h}$ of the finite-dimensional system

$$
\forall \boldsymbol{v}_{h} \in V_{h}, \quad a_{h}\left(\boldsymbol{u}_{h}, \boldsymbol{v}_{h}\right)=\int_{\Omega} \boldsymbol{f}(\boldsymbol{x}) \cdot \boldsymbol{v}_{h}(\boldsymbol{x}) \mathrm{d} \boldsymbol{x}
$$

Moreover, as already recalled from [8, Lemma 2], the form $a_{h}(\cdot, \cdot)$ is positive definite on $X_{h}$. So Problem (2.11) has a unique solution $\boldsymbol{u}_{h}$ in $V_{h}$.

Thanks to this result, the well-posedness of Problem (2.6) relies on the inf-sup condition that is established later on.

\section{Further REMARKS on the Discrete PRESSure SPACE}

We begin by checking the uniform continuity of the form $b_{h}(\cdot, \cdot)$ on the discrete spaces (here, "uniform" means that its norm is bounded independently of $h$ ). Throughout the paper, $c, c^{\prime}, c^{\prime \prime}$ stand for generic constants that may vary from a line to the next one but are always independent of $h$.

Lemma 3.1. There exists a constant $c$ independent of $h$ such that the following continuity property holds

$$
\forall \boldsymbol{v}_{h} \in X_{h}, \forall q_{h} \in M_{h}, \quad\left|b_{h}\left(\boldsymbol{v}_{h}, q_{h}\right)\right| \leq c\left\|\boldsymbol{v}_{h}\right\|_{H_{h}^{1}(\Omega)}\left\|q_{h}\right\|_{L^{2}(\Omega)} .
$$


Proof. Integrating by parts in the definition $(2.7)$ of $b_{h}(\cdot, \cdot)$ yields (we denote by $\boldsymbol{n}$ the unit outward normal to $K$ )

$$
b_{h}\left(\boldsymbol{v}_{h}, q_{h}\right)=\sum_{K \in \mathcal{T}_{h}}\left(-\int_{K} q_{h}\left(\operatorname{div} \boldsymbol{v}_{h}\right) \mathrm{d} \boldsymbol{x}+\int_{\partial K} \boldsymbol{v}_{h} \cdot \boldsymbol{n} q_{h} \mathrm{~d} \tau\right) .
$$

Bounding the first term is obvious:

$$
\left|\int_{K} q_{h}\left(\operatorname{div} \boldsymbol{v}_{h}\right) \mathrm{d} \boldsymbol{x}\right| \leq \sqrt{d}\left|\boldsymbol{v}_{h}\right|_{H^{1}(K)^{d}}\left\|q_{h}\right\|_{L^{2}(\Omega)} .
$$

As far as the second one is concerned, we consider an edge $(d=2)$ or face $(d=3) e$ in $\mathcal{E}_{h}$ and we agree to denote by $\bar{w}_{h}^{e}$ the mean value of any polynomial $w_{h}$ on $e$. Indeed, if $e$ is contained in $\partial \Omega$, since $\boldsymbol{v}_{h} \cdot \boldsymbol{n}$ is affine on $e$ and vanishes in the midpoint of $e$, we have

$$
\int_{e} \boldsymbol{v}_{h} \cdot \boldsymbol{n} q_{h} \mathrm{~d} \tau=\int_{e} \boldsymbol{v}_{h} \cdot \boldsymbol{n}\left(q_{h}-\bar{q}_{h}^{e}\right) \mathrm{d} \tau .
$$

If $e$ is not contained in $\partial \Omega$, the integral in $e$ appears twice in the previous sum on the $K$ in $\mathcal{T}_{h}$, so it can be replaced by $\frac{1}{2} \int_{e}\left[\boldsymbol{v}_{h} \cdot \boldsymbol{n}\right] q_{h} \mathrm{~d} \tau$ (here, [·] stands for the jump of a function through $e$ with appropriate sign) and, since now $\left[\boldsymbol{v}_{h} \cdot \boldsymbol{n}\right]$ is affine and vanishes in the midpoint of $e$,

$$
\frac{1}{2} \int_{e}\left[\boldsymbol{v}_{h} \cdot \boldsymbol{n}\right] q_{h} \mathrm{~d} \tau=\frac{1}{2} \int_{e}\left[\boldsymbol{v}_{h} \cdot \boldsymbol{n}\right]\left(q_{h}-\bar{q}_{h}^{e}\right) \mathrm{d} \tau .
$$

So, we have proven that

$$
\sum_{K \in \mathcal{T}_{h}} \int_{\partial K} \boldsymbol{v}_{h} \cdot \boldsymbol{n} q_{h} \mathrm{~d} \tau=\sum_{K \in \mathcal{T}_{h}} \sum_{e \subset \partial K} \int_{e} \boldsymbol{v}_{h} \cdot \boldsymbol{n}\left(q_{h}-\bar{q}_{h}^{e}\right) \mathrm{d} \tau .
$$

Next, on each edge $e$ in $\mathcal{E}_{h}$, we have

$$
\int_{e} \boldsymbol{v}_{h} \cdot \boldsymbol{n}\left(q_{h}-\bar{q}_{h}^{e}\right) \mathrm{d} \tau=\int_{e}\left(\boldsymbol{v}_{h}-\overline{\boldsymbol{v}}_{h}^{e}\right) \cdot \boldsymbol{n}\left(q_{h}-\bar{q}_{h}^{e}\right) \mathrm{d} \tau=\int_{e}\left(\boldsymbol{v}_{h}-\overline{\boldsymbol{v}}_{h}^{e}\right) \cdot \boldsymbol{n} q_{h} \mathrm{~d} \tau .
$$

Now, assume that $e$ is an edge or face of an element $K$ of $\mathcal{T}_{h}$. If $h_{K}$ denotes the diameter of $K$, we use the affine mapping that sends $K$ onto a reference element $\hat{K}$ and $e$ onto the edge or face $\hat{e}$ of $\hat{K}$ : with standard notation,

$$
\left|\int_{e} \boldsymbol{v}_{h} \cdot \boldsymbol{n}\left(q_{h}-\bar{q}_{h}^{e}\right) \mathrm{d} \tau\right| \leq c h_{K}^{d-1} \int_{\hat{e}}\left|\hat{\boldsymbol{v}}-\overline{\boldsymbol{v}}_{h}^{e}\right||\hat{q}| \mathrm{d} \hat{\tau}
$$

and using the equivalence of norms on the finite-dimensional space on the reference element yields

$$
\left|\int_{e} \boldsymbol{v}_{h} \cdot \boldsymbol{n}\left(q_{h}-\bar{q}_{h}^{e}\right) \mathrm{d} \tau\right| \leq c h_{K}^{d-1}\left\|\hat{\boldsymbol{v}}-\overline{\boldsymbol{v}}_{h}^{e}\right\|_{H^{1}(\hat{K})^{d}}\|\hat{q}\|_{L^{2}(\hat{K})} .
$$

Let $\hat{Y}_{\hat{e}}$ stand for the space

$$
\hat{Y}_{\hat{e}}=\left\{\hat{w} \in H^{1}(\hat{K}) ; \int_{\hat{e}} \hat{w}(\hat{\tau}) \mathrm{d} \hat{\tau}=0\right\}
$$

It is readily checked that the only function $\hat{w}$ in $\hat{Y}_{\hat{e}}$ that satisfies $|\hat{w}|_{H^{1}(\hat{K})}=0$ is zero. So, due to the compactness of $H^{1}(\hat{K})$ in $L^{2}(\hat{K})$, applying the Peetre-Tartar lemma [11, Chap. I, Th. 2.1] yields that the semi-norm $|\cdot|_{H^{1}(\hat{K})}$ 
is a norm on $\hat{Y}_{\hat{e}}$, equivalent to the norm $\|\cdot\|_{H^{1}(\hat{K})}$. Since the meanvalue of $\boldsymbol{v}_{h}$ on $e$ is the same as the mean-value of $\hat{\boldsymbol{v}}$ on $\hat{e}$, applying this result to $\hat{\boldsymbol{v}}-\overline{\boldsymbol{v}}_{h}^{e}$ which belongs to $\hat{Y}_{\hat{e}}^{d}$ gives

$$
\left|\int_{e} \boldsymbol{v}_{h} \cdot \boldsymbol{n}\left(q_{h}-\bar{q}_{h}^{e}\right) \mathrm{d} \tau\right| \leq c h_{K}^{d-1}|\hat{\boldsymbol{v}}|_{H^{1}(\hat{K})^{d}}\|\hat{q}\|_{L^{2}(\hat{K})} .
$$

Going back to the triangle $K$ yields

$$
\left|\int_{e} \boldsymbol{v}_{h} \cdot \boldsymbol{n}\left(q_{h}-\bar{q}_{h}^{e}\right) \mathrm{d} \tau\right| \leq c h_{K}^{d-1}\left(h_{K}^{1-\frac{d}{2}}\left|\boldsymbol{v}_{h}\right|_{H^{1}(K)^{d}}\right)\left(h_{K}^{-\frac{d}{2}}\left\|q_{h}\right\|_{L^{2}(K)}\right)=c\left|\boldsymbol{v}_{h}\right|_{H^{1}(K)^{d}}\left\|q_{h}\right\|_{L^{2}(K)}
$$

which, combined with (3.3) and a Cauchy-Schwarz inequality, leads to the desired result.

The proof of the inf-sup condition for the Crouzeix-Raviart element, where the space of velocities is exactly the same space $X_{h}$ and the space of pressures is made of piecewise constant functions, see [8, §6], relies on the following operator $\Pi_{h}$ : for any smooth enough vector field $\boldsymbol{v}$ vanishing on $\partial \Omega, \Pi_{h} \boldsymbol{v}$ belongs to $X_{h}$ and satisfies

$$
\forall e \in \mathcal{E}_{h}, \quad \int_{e} \Pi_{h} \boldsymbol{v} \mathrm{d} \tau=\int_{e} \boldsymbol{v} \mathrm{d} \tau
$$

It follows from the formula

$$
\operatorname{meas}(e)\left(\Pi_{h} \boldsymbol{v}\right)\left(\boldsymbol{m}_{e}\right)=\int_{e} \Pi_{h} \boldsymbol{v} \mathrm{d} \tau,
$$

that this operator is well-defined. In the following lemma, we state and briefly prove its main properties that are needed later on.

Lemma 3.2. The operator $\Pi_{h}$ is continuous on $H_{0}^{1}(\Omega)^{d}$. Moreover, there exists a constant $c$ independent of $h$ such that

$$
\forall \boldsymbol{v} \in H_{0}^{1}(\Omega)^{d}, \quad\left\|\Pi_{h} \boldsymbol{v}\right\|_{H_{h}^{1}(\Omega)} \leq c|\boldsymbol{v}|_{H^{1}(\Omega)^{d}},
$$

and

$$
\forall \boldsymbol{v} \in H_{0}^{1}(\Omega)^{d}, \forall K \in \mathcal{T}_{h}, \quad\left\|\boldsymbol{v}-\Pi_{h} \boldsymbol{v}\right\|_{L^{2}(K)^{d}} \leq c h_{K}|\boldsymbol{v}|_{H^{1}(K)^{d}} .
$$

Proof. Due to the standard trace theorem, the continuity of the operator $\Pi_{h}$ is clear. To go further, we fix a function $\boldsymbol{v}$ in $H_{0}^{1}(\Omega)^{d}$ and an element $K$ of $\mathcal{T}_{h}$, and we use the affine mapping that sends $K$ onto a reference element $\hat{K}$ : with standard notation and obvious local definition of the operator $\hat{\Pi}$,

$$
\left|\boldsymbol{v}-\Pi_{h} \boldsymbol{v}\right|_{H^{1}(K)^{d}} \leq c h_{K}^{\frac{d}{2}-1}|\hat{\boldsymbol{v}}-\hat{\Pi} \hat{\boldsymbol{v}}|_{H^{1}(\hat{K})^{d}} .
$$

We observe that the operator $\hat{\Pi}$ is equal to the identity on all affine functions on $\hat{K}$, hence on all constants, and also that it is continuous on $H^{1}(\hat{K})^{d}$ thanks to the trace theorem on $\hat{K}$. So, if $\hat{c}$ stands for its norm, we have

$$
\left|\boldsymbol{v}-\Pi_{h} \boldsymbol{v}\right|_{H^{1}(K)^{d}} \leq c h_{K}^{\frac{d}{2}-1} \inf _{\lambda \in \mathbb{R}}\|(I d-\hat{\Pi})(\hat{\boldsymbol{v}}-\lambda)\|_{H^{1}(\hat{K})^{d}} \leq c(1+\hat{c}) h_{K}^{\frac{d}{2}-1} \inf _{\lambda \in \mathbb{R}}\|\hat{\boldsymbol{v}}-\lambda\|_{H^{1}(\hat{K})^{d}} .
$$

Applying the Bramble-Hilbert inequality [6, Th. 14.1] and going back to the element $K$ gives

$$
\left|\boldsymbol{v}-\Pi_{h} \boldsymbol{v}\right|_{H^{1}(K)^{d}} \leq c^{\prime} h_{K}^{\frac{d}{2}-1}|\hat{\boldsymbol{v}}|_{H^{1}(\hat{K})^{d}} \leq c^{\prime \prime}|\boldsymbol{v}|_{H^{1}(K)^{d}},
$$


which yields (3.5). The proof of (3.6) is exactly the same, relying on the inequality

$$
\left\|\boldsymbol{v}-\Pi_{h} \boldsymbol{v}\right\|_{L^{2}(K)^{d}} \leq c h_{K}^{\frac{d}{2}}\|\hat{\boldsymbol{v}}-\hat{\Pi} \hat{\boldsymbol{v}}\|_{H^{1}(\hat{K})^{d}} .
$$

The next step consists in introducing a decomposition of both spaces $X_{h}$ and $M_{h}$ into two subspaces. With each edge or face $e$ of $\mathcal{E}_{h}$, we associate one of the unit normal vectors to $e$, which we denote by $\boldsymbol{n}_{e}$. This allows us to cut the space $X_{h}$ into two parts

$$
\begin{aligned}
& X_{h}^{T}=\left\{\boldsymbol{v}_{h} \in X_{h} ; \forall e \in \mathcal{E}_{h},\left(\boldsymbol{v}_{h} \cdot \boldsymbol{n}_{e}\right)\left(\boldsymbol{m}_{e}\right)=0\right\}, \\
& X_{h}^{N}=\left\{\boldsymbol{v}_{h} \in X_{h} ; \forall e \in \mathcal{E}_{h},\left(\boldsymbol{v}_{h} \times \boldsymbol{n}_{e}\right)\left(\boldsymbol{m}_{e}\right)=0\right\} .
\end{aligned}
$$

It is readily checked that $X_{h}$ is the direct sum of $X_{h}^{T}$ and $X_{h}^{N}$.

Similarly, we introduce the following spaces $M_{h}^{T}$ and $M_{h}^{N}$ :

$$
\begin{aligned}
M_{h}^{T} & =\left\{q_{h} \in H^{1}(\Omega) ; \forall K \in \mathcal{T}_{h}, q_{h \mid K} \in \mathcal{P}_{1}(K)\right\}, \\
M_{h}^{N} & =\operatorname{Span}\left\{\psi_{K} ; K \in \mathcal{T}_{h}\right\} .
\end{aligned}
$$

We denote by $M_{h}^{T 0}$ and $M_{h}^{N 0}$ the intersection of $L_{0}^{2}(\Omega)$ with $M_{h}^{T}$ and $M_{h}^{N}$, respectively. The degrees of freedom of the new spaces $X_{h}^{T}$ and $M_{h}^{T}$, resp. $X_{h}^{N}$ and $M_{h}^{N}$, are illustrated in the left, resp. right, part of the following figure (with arrows for the spaces $X_{h}^{T}$ and $X_{h}^{N}$, black dots for the spaces $M_{h}^{T}$ and $M_{h}^{N}$ ). The idea of this decomposition is explained in the next lemma.
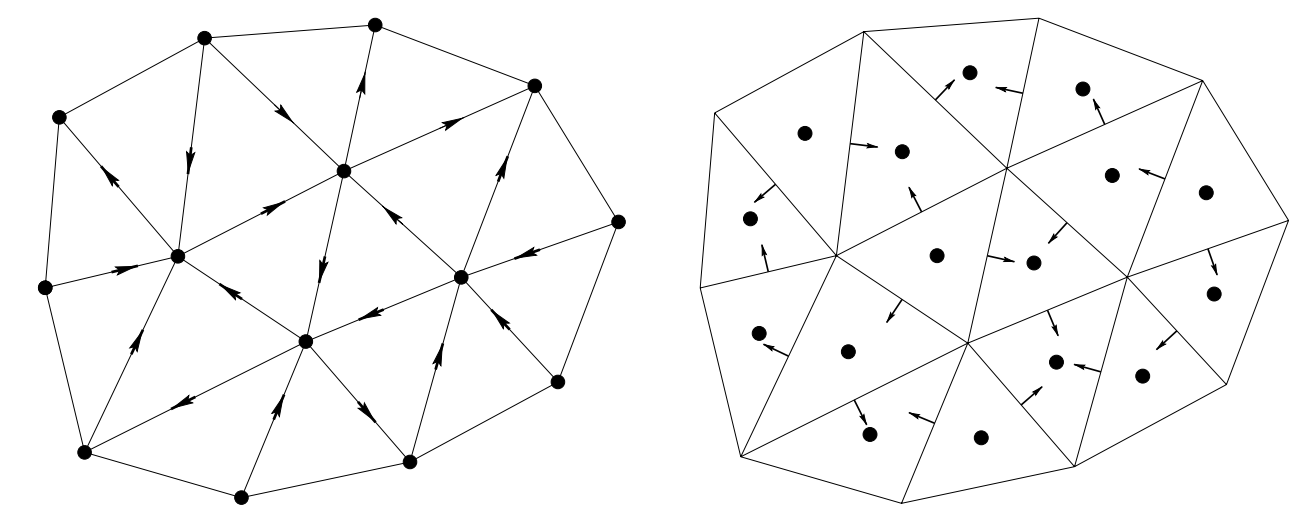

Figure 1.

Lemma 3.3. The following orthogonality property holds

$$
\forall \boldsymbol{v}_{h} \in X_{h}^{T}, \quad \forall q_{h} \in M_{h}^{N}, \quad b_{h}\left(\boldsymbol{v}_{h}, q_{h}\right)=0 .
$$

Proof. It suffices to check that, for all $K$ in $\mathcal{T}_{h}$,

$$
\forall \boldsymbol{v}_{h} \in X_{h}^{T}, \quad b_{h}\left(\boldsymbol{v}_{h}, \psi_{K}\right)=0 .
$$

So, let $K$ be any triangle in $\mathcal{T}_{h}$. Since the support of $\psi_{K}$ is $K$, it follows from the definition of $b_{h}(\cdot, \cdot)$ and by integration by parts that, for all $\boldsymbol{v}_{h}$ in $X_{h}^{T}$,

$$
b_{h}\left(\boldsymbol{v}_{h}, \psi_{K}\right)=\int_{K} \boldsymbol{v}_{h} \cdot \operatorname{grad} \psi_{K} \mathrm{~d} \boldsymbol{x}=-\int_{K} \psi_{K}\left(\operatorname{div} \boldsymbol{v}_{h}\right) \mathrm{d} \boldsymbol{x} .
$$


Next, we observe that $\operatorname{div} \boldsymbol{v}_{h}$ is constant on each $K$. Moreover, by integration by parts, we have (with appropriate choice of the directions of the $\boldsymbol{n}_{e}$ )

$$
\left(\operatorname{div} \boldsymbol{v}_{h}\right)_{\mid K} \operatorname{meas}(K)=\int_{K} \operatorname{div} \boldsymbol{v}_{h} \mathrm{~d} \boldsymbol{x}=\sum_{e \subset \partial K} \int_{e} \boldsymbol{v}_{h} \cdot \boldsymbol{n}_{e} \mathrm{~d} \boldsymbol{x}
$$

Since $\boldsymbol{v}_{h} \cdot \boldsymbol{n}_{e}$ is affine on each edge of $\partial K$ and vanishes in the midpoint of this edge from the definition of $X_{h}^{T}$, these integrals are equal to zero. So, $\left(\operatorname{div} \boldsymbol{v}_{h}\right)_{\mid K}$ is zero, which implies the desired orthogonality property.

Remark. As a by-product of the previous proof, we have checked that all functions of $X_{h}^{T}$ are divergence-free on each element $K$ of $\mathcal{T}_{h}$.

Let us recall from $[6,(25.14)]$ two formulas that will be of great use in what follows (they can also be proven by going to a reference element):

$$
\int_{K} \psi_{K} \mathrm{~d} \boldsymbol{x}=\frac{d !}{(2 d+1) !} \operatorname{meas}(K), \quad \int_{K} \psi_{K}^{2} \mathrm{~d} \boldsymbol{x}=\frac{2^{d+1} d !}{(3 d+2) !} \operatorname{meas}(K) .
$$

We now exhibit "spurious" modes, separately in $M_{h}^{T}$ and in $M_{h}^{N}$.

Lemma 3.4. The space of functions $q_{h}$ in $M_{h}^{T}$ such that

$$
\forall \boldsymbol{v}_{h} \in X_{h}, \quad b_{h}\left(\boldsymbol{v}_{h}, q_{h}\right)=0,
$$

contains the function 1 . The space of functions $q_{h}$ in $M_{h}^{N}$ satisfying (3.11) contains the function $\psi_{h}$ defined by

$$
\psi_{h}=\sum_{K \in \mathcal{T}_{h}} \psi_{K}
$$

Proof. From the definition of $b_{h}(\cdot, \cdot)$, it is obvious that the function 1 satisfies (3.11). So, we now consider the function $\psi_{h}$ and, since it vanishes on all the $\partial K, K \in \mathcal{T}_{h}$, we have for any $\boldsymbol{v}_{h}$ in $X_{h}$

$$
b\left(\boldsymbol{v}_{h}, \psi_{h}\right)=-\sum_{K \in \mathcal{T}_{h}}\left(\operatorname{div} \boldsymbol{v}_{h}\right)_{\mid K} \int_{K} \psi_{K} \mathrm{~d} \boldsymbol{x} .
$$

Using (3.10) gives

$$
b\left(\boldsymbol{v}_{h}, \psi_{h}\right)=-\frac{d !}{(2 d+1) !} \sum_{K \in \mathcal{T}_{h}} \int_{K} \operatorname{div} \boldsymbol{v}_{h} \mathrm{~d} \boldsymbol{x}=-\frac{d !}{(2 d+1) !} \sum_{K \in \mathcal{T}_{h}} \sum_{e \subset \partial K} \int_{e} \boldsymbol{v}_{h} \cdot \boldsymbol{n}_{e} \mathrm{~d} \tau .
$$

The previous sum can be rewritten as a sum on all edges in dimension $d=2$, faces in dimension $d=3$, of the $K$. Such an edge or face $e$ is either contained in $\partial \Omega$ or in the intersection of two elements $K$ : in the first case, $\boldsymbol{v}_{h} \cdot \boldsymbol{n}_{e}$ is affine and vanishes in the midpoint of $e$, in the second case the jump $\left[\boldsymbol{v}_{h} \cdot \boldsymbol{n}_{e}\right]$ that appears in the integral is affine and vanishes in the midpoint of $e$. So in all cases, the integral on $e$ is equal to zero, which yields

$$
b\left(\boldsymbol{v}_{h}, \psi_{h}\right)=0
$$

Fortunately, neither the function 1 nor $\psi_{h}$ belongs to $L_{0}^{2}(\Omega)$. So, we decide to work with the subspace $M_{h}^{0}$ defined by

$$
M_{h}^{0}=M_{h}^{T 0} \oplus M_{h}^{N 0}
$$


The idea is now to prove inf-sup conditions first between the spaces $X_{h}^{T}$ and $M_{h}^{T 0}$, second between $X_{h}^{N}$ and $M_{h}^{N 0}$. As a by-product, these inf-sup conditions imply that the spurious modes exhibited in Lemma 3.4 are the only ones.

Remark. From (3.10) and [6, (25.14)], it can be checked that

$$
\forall q_{h}^{T} \in M_{h}^{T}, \forall q_{h}^{N} \in M_{h}^{N}, \quad \int_{\Omega} q_{h}^{T} q_{h}^{N} \mathrm{~d} \boldsymbol{x} \leq\left(\frac{d !(3 d+2) !}{2^{d+1}(2 d+1) !^{2}}\right)^{\frac{1}{2}}\left\|q_{h}^{T}\right\|_{L^{2}(\Omega)}\left\|q_{h}^{N}\right\|_{L^{2}(\Omega)} .
$$

This means that the angle between $M_{h}^{T}$ and $M_{h}^{N}$ in $L^{2}(\Omega)$ is larger than a parameter $\kappa$ independent of $h$. In contrast, the intersection of $X_{h}^{T}$ and $X_{h}^{N}$ is reduced to $\{0\}$, however the angle between them for the scalar product associated with the norm $\|\cdot\|_{H_{h}^{1}(\Omega)}$ may tend to zero with $h$. Indeed, the function $\boldsymbol{v}_{h}^{T}+\boldsymbol{v}_{h}^{N}$ equal to 1 in each midpoint of an edge or barycenter of a face internal to $\Omega$ satisfies

$$
\inf \left\{\left\|\boldsymbol{v}_{h}^{T}\right\|_{H_{h}^{1}(\Omega)},\left\|\boldsymbol{v}_{h}^{N}\right\|_{H_{h}^{1}(\Omega)}\right\} \geq c h^{-1}\left(\inf _{K \in \mathcal{T}_{h}} h_{K}\right)^{\frac{1}{2}}\left\|\boldsymbol{v}_{h}^{T}+\boldsymbol{v}_{h}^{N}\right\|_{H_{h}^{1}(\Omega)},
$$

whence

$$
\left|\sum_{K \in \mathcal{T}_{h}} \int_{K} \operatorname{grad} \boldsymbol{v}_{h}^{T} \cdot \operatorname{grad} \boldsymbol{v}_{h}^{N} \mathrm{~d} \boldsymbol{x}\right| \geq\left(1-c^{2} h^{2}\left(\inf _{K \in \mathcal{T}_{h}} h_{K}\right)^{-1}\right)\left\|\boldsymbol{v}_{h}^{T}\right\|_{H_{h}^{1}(\Omega)}\left\|\boldsymbol{v}_{h}^{N}\right\|_{H_{h}^{1}(\Omega)} .
$$

\section{THE INF-SUP CONDITION FOR THE FIRST PAIR OF REDUCED SPACES}

We first prove an inf-sup condition between $X_{h}^{T}$ and $M_{h}^{T 0}$, where the constant is not independent of $h$. It requires a further assumption on the triangulation $\mathcal{T}_{h}$ that we now state.

Hypothesis 4.1. The boundary $\partial \Omega$ contains at most one edge in dimension $d=2$, at most two faces in dimension $d=3$, of the same element $K$ in $\mathcal{T}_{h}$.

The proof of the next lemma is very similar to that used for proving the inf-sup condition for the Taylor-Hood element, see [11, Chap. II, Th. 4.2].

Lemma 4.2. If Hypothesis 4.1 holds, there exists a constant $\beta_{T 0}$ independent of $h$ such that the following inf-sup condition holds

$$
\forall q_{h} \in M_{h}^{T 0}, \quad \sup _{\boldsymbol{v}_{h} \in X_{h}^{T}} \frac{b_{h}\left(\boldsymbol{v}_{h}, q_{h}\right)}{\left\|\boldsymbol{v}_{h}\right\|_{H_{h}^{1}(\Omega)}} \geq \beta_{T 0}\left(\inf _{K \in \mathcal{T}_{h}} h_{K}\right)\left\|q_{h}\right\|_{L^{2}(\Omega)} .
$$

Proof. Let $q_{h}$ be any element in $M_{h}^{T 0}$. From the definition of $M_{h}^{T}$, $\operatorname{grad} q_{h}$ is constant on each element $K$, so that for all $\boldsymbol{v}_{h}$ in $X_{h}^{T}$,

$$
b_{h}\left(\boldsymbol{v}_{h}, q_{h}\right)=\sum_{K \in \mathcal{T}_{h}}\left(\operatorname{grad} q_{h}\right)_{\mid K} \cdot \int_{K} \boldsymbol{v}_{h} \mathrm{~d} \boldsymbol{x} .
$$

Let us recall that, for any function $w$ in $\mathcal{P}_{1}(K)$,

$$
\int_{K} w \mathrm{~d} \boldsymbol{x}=\frac{\operatorname{meas}(K)}{d+1} \sum_{e \subset \partial K} w\left(\boldsymbol{m}_{e}\right)
$$

This gives

$$
b_{h}\left(\boldsymbol{v}_{h}, q_{h}\right)=\sum_{K \in \mathcal{T}_{h}} \frac{\operatorname{meas}(K)}{d+1}\left(\operatorname{grad} q_{h}\right)_{\mid K} \cdot \sum_{e \subset \partial K, e \not \supset \partial \Omega} \boldsymbol{v}_{h}\left(\boldsymbol{m}_{e}\right) .
$$


Since all $\boldsymbol{v}_{h} \cdot \boldsymbol{n}_{e}$ vanish in $\boldsymbol{m}_{e}$ from the definition of $X_{h}^{T}$ and since the tangential part of $\operatorname{grad} q_{h}$ is continuous through each $e$, the idea is to take

$$
\left(\boldsymbol{v}_{h} \times \boldsymbol{n}_{e}\right)\left(\boldsymbol{m}_{e}\right)=\left(\operatorname{grad} q_{h}\right)_{\mid K} \times \boldsymbol{n}_{e},
$$

which yields

$$
b_{h}\left(\boldsymbol{v}_{h}, q_{h}\right)=\sum_{K \in \mathcal{T}_{h}} \frac{\operatorname{meas}(K)}{d+1} \sum_{e \subset \partial K, e \not \subset \partial \Omega}\left|\left(\operatorname{grad} q_{h}\right)_{\mid K} \times \boldsymbol{n}_{e}\right|^{2} .
$$

Thanks to Hypothesis 4.1, in the case of dimension $d=2$, at least two edges of the same triangle $K$ are not contained in $\partial \Omega$, so that the unit tangential vectors to these edges form a basis of $\mathbb{R}^{2}$; moreover the angle between them belongs to an interval $] \eta, \pi-\eta[$, where $\eta$ only depends on the parameter $\sigma$ that measures the regularity of the family $\left(\mathcal{T}_{h}\right)_{h}$. Similarly, in dimension $d=3$, at least two faces $e$ and $e^{\prime}$ of $K$ are not contained in $\partial \Omega$. The unit vector $\boldsymbol{\tau}$ on their common edge and the vectors orthogonal to $\tau$ in $e$ and $e^{\prime}$ form a basis of $\mathbb{R}^{3}$ and the angle between $e$ and $e^{\prime}$ also belongs to an interval $] \eta, \pi-\eta[$ which is analogous to the previous one. So, we obtain

$$
b_{h}\left(\boldsymbol{v}_{h}, q_{h}\right) \geq c \sum_{K \in \mathcal{T}_{h}} \operatorname{meas}(K)\left|\left(\operatorname{grad} q_{h}\right)_{\mid K}\right|^{2},
$$

or, equivalently,

$$
b_{h}\left(\boldsymbol{v}_{h}, q_{h}\right) \geq c\left|q_{h}\right|_{H^{1}(\Omega)}^{2},
$$

where the constant $c$ only depends on $\sigma$. Next, using the Lagrange function $\mu_{e}$ which belongs to $\mathcal{P}_{1}(K)$ for all $K$ in $\mathcal{T}_{h}$ and vanishes in all the midpoints of the edges, respectively the barycenters of the faces, of elements $K$ in $\mathcal{T}_{h}$ but in $\boldsymbol{m}_{e}$ where it is equal to 1 , we derive by going to a reference element that

$$
\left|\mu_{e}\right|_{H^{1}(K)} \leq c h_{e}^{\frac{d}{2}-1}
$$

where $h_{e}$ stands for the diameter of $e$. This yields

$$
\left|\boldsymbol{v}_{h}\right|_{H^{1}(K)} \leq c h_{K}^{-1} \operatorname{meas}(K)^{\frac{1}{2}}\left|\left(\operatorname{grad} q_{h}\right)_{\mid K}\right|,
$$

whence

$$
\left\|\boldsymbol{v}_{h}\right\|_{H_{h}^{1}(\Omega)} \leq c\left(\inf _{K \in \mathcal{T}_{h}} h_{K}\right)^{-1}\left|q_{h}\right|_{H^{1}(\Omega)} .
$$

Due to this inequality, we derive from (4.2) that

$$
\sup _{\boldsymbol{v}_{h} \in X_{h}^{T}} \frac{b_{h}\left(\boldsymbol{v}_{h}, q_{h}\right)}{\left\|\boldsymbol{v}_{h}\right\|_{H_{h}^{1}(\Omega)}} \geq c\left(\inf _{K \in \mathcal{T}_{h}} h_{K}\right)\left|q_{h}\right|_{H^{1}(\Omega)} .
$$

By combining this estimate with the standard Bramble-Hilbert inequality [6, Th. 14.1]

$$
\forall q \in L_{0}^{2}(\Omega), \quad\|q\|_{L^{2}(\Omega)} \leq c|q|_{H^{1}(\Omega)},
$$

we obtain the desired result.

Remark. Let us associate with any vertex $\boldsymbol{a}$ of an element in $\mathcal{T}_{h}$ the union $\bar{\Omega}_{\boldsymbol{a}}$ of all elements $K$ in $\mathcal{T}_{h}$ that contain $\boldsymbol{a}$. We recall [11, Chap. I, §A.3] that there exists a finite number of reference subdomains such that each $\Omega_{\boldsymbol{a}}$ can be mapped onto one of them by a continuous piecewise affine mapping. So a scaled Bramble-Hilbert inequality on the $\Omega_{a}$ can be derived by going to the reference subdomain. Thus, if Hypothesis 4.1 holds, the same arguments as previously lead to an optimal inf-sup condition (i.e. with constant independent of $h$ ) between 
the space $X_{\boldsymbol{a}}^{T}$ of restrictions to $\Omega_{\boldsymbol{a}}$ of functions in $X_{h}^{T}$ which vanish in the midpoints of the edges contained in $\partial \Omega_{\boldsymbol{a}}$ and the space $M_{\boldsymbol{a}}^{T 0}=M_{\boldsymbol{a}}^{T} \cap L_{0}^{2}\left(\Omega_{\boldsymbol{a}}\right)$, where $M_{\boldsymbol{a}}^{T}$ denote the space of restrictions of functions in $M_{h}^{T}$ to $\Omega_{\boldsymbol{a}}$.

Remark. The constant in the inf-sup condition (4.1) is not independent of $h$ and exhibiting a counter-example to prove the optimality of this condition in the case of triangular finite elements seems difficult. However, let us for a while consider the bidimensional case of a rectangle $\Omega=]-1,1[\times] 0,1[$, provided with a "quadrangulation" made of $2 N^{2}$ equal squares with edges parallel to the coordinate axes, as illustrated in Figure 2 (the length of each edge is $\left.\frac{h}{\sqrt{2}}=N^{-1}\right)$. Let also $\hat{K}$ stand for the reference square with vertices $\left(\frac{1}{\sqrt{2}}, 0\right),\left(\frac{2}{\sqrt{2}}, \frac{1}{\sqrt{2}}\right),\left(\frac{1}{\sqrt{2}}, \frac{2}{\sqrt{2}}\right)$ and $\left(0, \frac{1}{\sqrt{2}}\right)$. We consider the space $X_{h}^{T}$ defined as previously, except that $\mathcal{P}_{1}(K)$ is replaced by the space

$$
\left\{\hat{v} \circ F_{K}^{-1} ; \hat{v} \in \mathcal{Q}_{1}(\hat{K})\right\}
$$

where $\mathcal{Q}_{1}(\hat{K})$ stands for the space of functions on $\hat{K}$ which are affine with respect to each variable and $F_{K}$ is one of the mappings in $\mathcal{Q}_{1}(\hat{K})^{2}$ that maps $\hat{K}$ onto $K$. We work with the function $q_{h}$ equal to the first coordinate $x$, which belongs to $L_{0}^{2}(\Omega)$. Then, the best inf-sup constant $\beta_{h}^{T}$ between $X_{h}^{T}$ and any space containing $q_{h}$ is smaller than the ratio $\left\|\boldsymbol{w}_{h}\right\|_{H_{h}^{1}(\Omega)} /\left\|q_{h}\right\|_{L^{2}(\Omega)}$, where $\boldsymbol{w}_{h}=\left(w_{h x}, w_{h y}\right)$ is the only solution inf $X_{h}^{T}$ of the problem

$$
\forall \boldsymbol{v}_{h}=\left(v_{h x}, v_{h y}\right) \in X_{h}^{T}, \quad a_{h}\left(\boldsymbol{w}_{h}, \boldsymbol{v}_{h}\right)=b_{h}\left(\boldsymbol{v}_{h}, q_{h}\right)=\int_{\Omega} v_{h x}(\boldsymbol{x}) \mathrm{d} \boldsymbol{x} .
$$

It can be checked that this solution satisfies $w_{h y}=0$ and also that $w_{h x}$ vanishes on the midpoints of all edges of the $K$ which are parallel to the $y$-axis. So, by applying a scaled Poincaré-Friedrichs inequality on each square $K$ (derived by going to the reference element), we can prove that $\beta_{h}^{T}$ is smaller than $c h$. In view of this counter-example, we think that the constant in (4.1) is of best possible order.

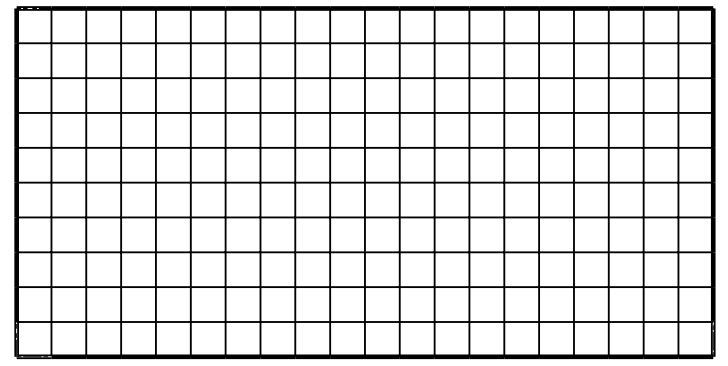

FIGURE 2 .

Even if the inf-sup condition (4.1) is not optimal, it provides the following result.

Corollary 4.3. If Hypothesis 4.1 holds, the space of functions $q_{h}$ in $M_{h}^{T}$ such that

$$
\forall \boldsymbol{v}_{h} \in X_{h}^{T}, \quad b_{h}\left(\boldsymbol{v}_{h}, q_{h}\right)=0,
$$

has dimension 1 and is spanned by the function 1.

Remark. Hypothesis 4.1 is not very restrictive, and it is necessary for the assertion of Corollary 4.3 to be true. Indeed, consider the case where the domain $\Omega$ is a hexagon and a mesh $\mathcal{T}_{h}$ is built by joining one vertex out of two of the hexagon (see the next figure). In this situation, the space $M_{h}^{T}$ has dimension 6 , while the space $X_{h}^{T}$ has dimension 3. So, Corollary 4.3 does not hold. This counter-example can be extended to much more general geometries and meshes, built from the previous one by adding iteratively one exterior point and joining this point to the two endpoints of one edge of the boundary. 


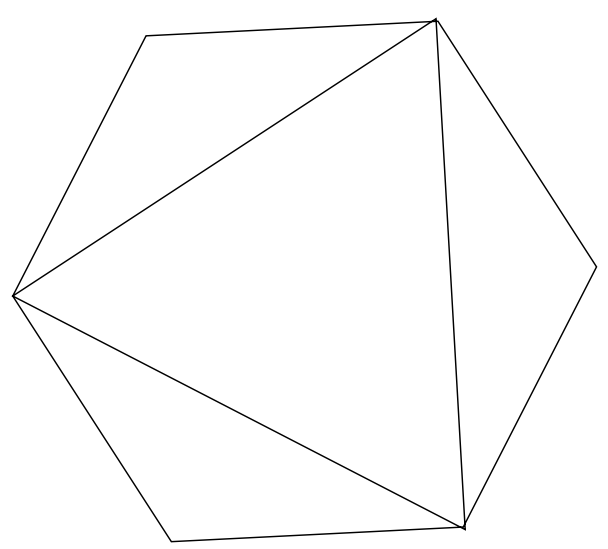

FiguRE 3 .

Next, we prove a slightly different version of Lemma 4.2 by very similar arguments.

Lemma 4.4. If Hypothesis 4.1 holds, there exists a constant $\beta_{T 0}$ independent of $h$ such that the following inf-sup condition holds

$$
\forall q_{h} \in M_{h}^{T 0}, \quad \sup _{\boldsymbol{v}_{h} \in X_{h}^{T}} \frac{b_{h}\left(\boldsymbol{v}_{h}, q_{h}\right)}{\left\|\boldsymbol{v}_{h}\right\|_{H_{h}^{1}(\Omega)}} \geq \beta_{T 0}\left(\sum_{K \in \mathcal{T}_{h}} h_{K}^{2}\left|q_{h}\right|_{H^{1}(K)}^{2}\right)^{\frac{1}{2}}
$$

Proof. Following the same lines as in the previous proof, we now choose the function $\boldsymbol{v}_{h}$ in $X_{h}^{T}$ which satisfies

$$
\left(\boldsymbol{v}_{h} \times \boldsymbol{n}_{e}\right)\left(\boldsymbol{m}_{e}\right)=h_{e}^{2}\left(\operatorname{grad} q_{h}\right)_{\mid K} \times \boldsymbol{n}_{e},
$$

where $h_{e}$ stands for the diameter of $e$. It follows from the regularity of the family of triangulations that, for each $K$ in $\mathcal{T}_{h}$ containing $e$,

$$
\sigma^{-1} h_{K} \leq h_{e} \leq h_{K}
$$

So, by the same arguments as previously, we derive

$$
b_{h}\left(\boldsymbol{v}_{h}, q_{h}\right) \geq c\left(\sum_{K \in \mathcal{T}_{h}} h_{K}^{2}\left|q_{h}\right|_{H^{1}(K)}^{2}\right)
$$

and

whence

$$
\left|\boldsymbol{v}_{h}\right|_{H^{1}(K)} \leq c h_{K} \operatorname{meas}(K)^{\frac{1}{2}}\left|\left(\operatorname{grad} q_{h}\right)_{\mid K}\right|,
$$

This yields the desired result.

$$
\left\|\boldsymbol{v}_{h}\right\|_{H_{h}^{1}(\Omega)} \leq c\left(\sum_{K \in \mathcal{T}_{h}} h_{K}^{2}\left|q_{h}\right|_{H^{1}(K)}^{2}\right)^{\frac{1}{2}}
$$

The inf-sup condition (4.5) does not involve the right-norm on $q_{h}$, but the mesh-dependent semi-norm

$$
\left\|q_{h}\right\|_{L_{h}^{2}(\Omega)}=\left(\sum_{K \in \mathcal{T}_{h}} h_{K}^{2}\left|q_{h}\right|_{H^{1}(K)}^{2}\right)^{\frac{1}{2}}
$$


It is readily checked that the quantity $\|\cdot\|_{L_{h}^{2}(\Omega)}$ is a norm on $M_{h}^{T 0}$ and also, by using the standard inverse inequality, that the following equivalence property holds

$$
\forall q_{h} \in M_{h}^{T 0}, \quad c_{h}\left\|q_{h}\right\|_{L^{2}(\Omega)} \leq\left\|q_{h}\right\|_{L_{h}^{2}(\Omega)} \leq c\left\|q_{h}\right\|_{L^{2}(\Omega)},
$$

where the constant $c$ does not depend on $h$. Unfortunately, the largest possible constant $c_{h}$ is not independent of $h$, but satisfies

$$
c\left(\inf _{K \in \mathcal{T}_{h}} h_{K}\right) \leq c_{h} \leq c^{\prime} h
$$

(the first inequality follows from Bramble-Hilbert one, the second one is obtained by taking $q_{h}$ equal to a global affine function).

We now prove an optimal inf-sup condition between $X_{h}$ and $M_{h}^{T 0}$, which relies on condition (4.5) combined with Verfürth's argument [14].

Proposition 4.5. If Hypothesis 4.1 holds, there exists a constant $\beta_{T}$ independent of $h$ such that the following inf-sup condition holds

$$
\forall q_{h} \in M_{h}^{T 0}, \quad \sup _{\boldsymbol{v}_{h} \in X_{h}} \frac{b_{h}\left(\boldsymbol{v}_{h}, q_{h}\right)}{\left\|\boldsymbol{v}_{h}\right\|_{H_{h}^{1}(\Omega)}} \geq \beta_{T}\left\|q_{h}\right\|_{L^{2}(\Omega)}
$$

Proof. Let $q_{h}$ be any function of $M_{h}^{T 0}$. Since $q_{h}$ belongs to $L_{0}^{2}(\Omega)$, it follows from $(2.5)$ that there exists a function $\boldsymbol{v}$ in $H_{0}^{1}(\Omega)^{d}$ such that

$$
\operatorname{div} \boldsymbol{v}=-q_{h} \quad \text { and } \quad|\boldsymbol{v}|_{H^{1}(\Omega)^{d}} \leq \frac{1}{\beta}\left\|q_{h}\right\|_{L^{2}(\Omega)}
$$

The idea is now to take the function $\boldsymbol{v}_{h}$ equal to $\Pi_{h} \boldsymbol{v}$, where the operator $\Pi_{h}$ is defined by (3.4). Indeed, we write

$$
b_{h}\left(\boldsymbol{v}_{h}, q_{h}\right)=b_{h}\left(\boldsymbol{v}, q_{h}\right)-b_{h}\left(\boldsymbol{v}-\Pi_{h} \boldsymbol{v}, q_{h}\right) .
$$

The first term is evaluated from (4.9) by integration by parts:

$$
b_{h}\left(\boldsymbol{v}, q_{h}\right)=\left\|q_{h}\right\|_{L^{2}(\Omega)}^{2} .
$$

Next, we have thanks to the Cauchy-Schwarz inequality

$$
\left|b_{h}\left(\boldsymbol{v}-\Pi_{h} \boldsymbol{v}, q_{h}\right)\right| \leq \sum_{K \in \mathcal{T}_{h}}\left\|\boldsymbol{v}-\Pi_{h} \boldsymbol{v}\right\|_{L^{2}(K)}\left|q_{h}\right|_{H^{1}(K)},
$$

and using (3.6) yields

$$
\left|b_{h}\left(\boldsymbol{v}-\Pi_{h} \boldsymbol{v}, q_{h}\right)\right| \leq c|\boldsymbol{v}|_{H^{1}(\Omega)^{d}}\left\|q_{h}\right\|_{L_{h}^{2}(\Omega)} .
$$

It follows from (3.5) and (4.9) that

$$
\left\|\Pi_{h} \boldsymbol{v}\right\|_{H_{h}^{1}(\Omega)} \leq c|\boldsymbol{v}|_{H^{1}(\Omega)^{d}} \leq \frac{c}{\beta}\left\|q_{h}\right\|_{L^{2}(\Omega)} .
$$

So, we derive

$$
\sup _{\boldsymbol{v}_{h} \in X_{h}} \frac{b_{h}\left(\boldsymbol{v}_{h}, q_{h}\right)}{\left\|\boldsymbol{v}_{h}\right\|_{H_{h}^{1}(\Omega)}} \geq c_{1}\left\|q_{h}\right\|_{L^{2}(\Omega)}-c_{2}\left\|q_{h}\right\|_{L_{h}^{2}(\Omega)},
$$


where the constants $c_{1}$ and $c_{2}$ are independent of $h$. Next, Verfürth's trick consists in comparing this estimate with (4.5) and noting that

$$
\forall t \geq 0, \quad \sup \left\{\beta_{T 0} t, c_{1}\left\|q_{h}\right\|_{L^{2}(\Omega)}-c_{2} t\right\} \geq \frac{\beta_{T 0} c_{1}}{\beta_{T 0}+c_{2}}\left\|q_{h}\right\|_{L^{2}(\Omega)},
$$

which gives the desired inf-sup condition.

\section{ThE INF-SUP CONDITION FOR THE SECOND PAIR OF REDUCED SPACES}

We are now interested in proving the inf-sup condition between the spaces $X_{h}^{N}$ and $M_{h}^{N 0}$. However, we prefer to begin with a slightly different space of pressures, namely

$$
M_{h}^{N *}=\left\{q_{h} \in M_{h}^{N} ; \int_{\Omega} q_{h} \psi_{h} \mathrm{~d} \boldsymbol{x}=0\right\},
$$

where $\psi_{h}$ is the function introduced in (3.12).

Our proof relies on the graph theory. We refer to [3] for the general notion of graphs and trees, and to [13] for its application to finite element meshes. We only give a very restricted definition. A tree $T_{h}$ associated with a triangulation $\mathcal{T}_{h}$ is a finite family of segments such that:

- the first segment starts from a point on $\partial \Omega$ (the root of the tree), more precisely from the midpoint of an edge or barycenter of a face of an element $K$ in $\mathcal{T}_{h}$ which is contained in $\partial \Omega$ and goes to the barycenter of this same element $K$;

- at each barycenter of an element $K$ in $\mathcal{T}_{h}$, either the tree stops or at most $d$ lines start from this barycenter and go to the barycenters of adjacent elements $K^{\prime}$ in $\mathcal{T}_{h}$ by crossing the midpoint or barycenter of the common edge or face of $K$ and $K^{\prime}$;

- the graph contains no cycle;

- each element $K$ in $\mathcal{T}_{h}$ intersects the graph.

This definition is illustrated in the following figure. We refer to [13] for slightly different but equivalent definitions.

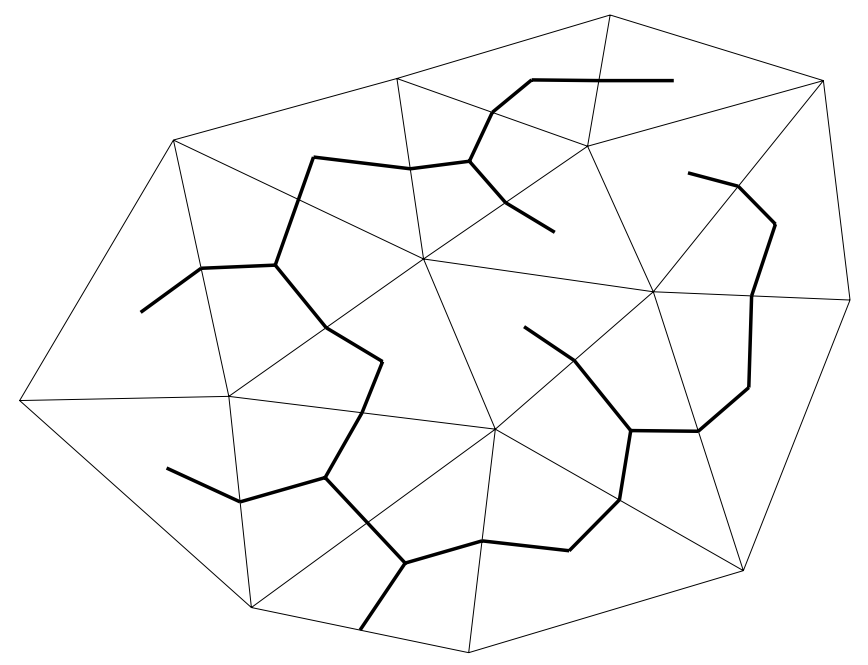

FiguRE 4.

The existence of such a tree for any triangulation $\mathcal{T}_{h}$ is proven in [13]. For completeness, we sketch the proof. 
Lemma 5.1. For any triangulation $\mathcal{T}_{h}$ of $\Omega$, there exists a tree associated with $\mathcal{T}_{h}$.

Proof. Let $\mathcal{T}_{h}$ be any triangulation of $\Omega$, and let $N_{h}$ be the number of elements of $\mathcal{T}_{h}$. We recall [13] that, since $\Omega$ is connected, the elements $K_{1}, \ldots, K_{N_{h}}$ of $\mathcal{T}_{h}$ can be numbered so that $K_{1} \cap \partial \Omega$ is an edge $(d=2)$ or a face $(d=3)$ of $K_{1}$ and that, for each $n, 1 \leq n \leq N_{h}$, the interior $\Omega_{n}$ of $\cup_{m=1}^{n} K_{m}$ is connected. The proof now relies on an induction argument over $n$.

1) If $n$ is equal to 1 , the tree associated with the triangulation $\left\{K_{1}\right\}$ is trivial.

2) Assume that there exists a tree associated with the triangulation $\left\{K_{1}, \ldots, K_{n-1}\right\}$ of $\Omega_{n-1}$ and consider the element $K_{n}$. There exists an element $K_{m}, 1 \leq m \leq n-1$, such that $K_{n}$ and $K_{m}$ share an edge or a face. Then the tree can be extended to $\left\{K_{1}, \ldots, K_{n}\right\}$ by adding the line which goes from the barycenter of $K_{m}$ to the barycenter of $K_{n}$.

This ends the proof.

We are now in a position to prove an inf-sup condition between $X_{h}^{N}$ and $M_{h}^{N *}$.

Lemma 5.2. There exists a constant $\beta_{N *}$ independent of $h$ such that the following inf-sup condition holds

$$
\forall q_{h} \in M_{h}^{N *}, \quad \sup _{\boldsymbol{v}_{h} \in X_{h}^{N}} \frac{b_{h}\left(\boldsymbol{v}_{h}, q_{h}\right)}{\left\|\boldsymbol{v}_{h}\right\|_{H_{h}^{1}(\Omega)}} \geq \frac{\beta_{N *}}{\operatorname{meas}(\Omega)}\left(\inf _{K \in \mathcal{T}_{h}} h_{K}\right)^{d}\left\|q_{h}\right\|_{L^{2}(\Omega)} .
$$

Proof. Let $q_{h}$ be any element in $M_{h}^{N *}$. We wish to exhibit a function $\boldsymbol{v}_{h}$ in $X_{h}^{N}$ such that

$$
\forall r_{h} \in M_{h}^{N}, \quad b_{h}\left(\boldsymbol{v}_{h}, r_{h}\right)=\int_{\Omega} q_{h} r_{h} \mathrm{~d} \boldsymbol{x} .
$$

From the definition of $M_{h}^{N}$, this is equivalent to

$$
\forall K \in \mathcal{T}_{h}, \quad \int_{K} \boldsymbol{v}_{h} \cdot \operatorname{grad} \psi_{K} \mathrm{~d} \boldsymbol{x}=\int_{K} q_{h} \psi_{K} \mathrm{~d} \boldsymbol{x},
$$

hence, by integration by parts, to

$$
\forall K \in \mathcal{T}_{h}, \quad-\left(\operatorname{div} \boldsymbol{v}_{h}\right)_{\mid K} \int_{K} \psi_{K} \mathrm{~d} \boldsymbol{x}=\int_{K} q_{h} \psi_{K} \mathrm{~d} \boldsymbol{x} .
$$

Next, applying the Stokes' formula gives (the orientation of the $\boldsymbol{n}_{e}$ is implicit throughout this proof)

$$
\operatorname{meas}(K)\left(\operatorname{div} \boldsymbol{v}_{h}\right)_{\mid K}=\int_{K} \operatorname{div} \boldsymbol{v}_{h} \mathrm{~d} \boldsymbol{x}=\sum_{e \in \mathcal{E}_{h}, e \subset \partial K} \operatorname{meas}(e)\left(\boldsymbol{v}_{h} \cdot \boldsymbol{n}_{e}\right)\left(\boldsymbol{m}_{e}\right) .
$$

Moreover, if $\alpha$ denotes the constant $\frac{d !}{(2 d+1) !}$, we know from (3.10) that

$$
\int_{K} \psi_{K} \mathrm{~d} \boldsymbol{x}=\alpha \operatorname{meas}(K)
$$

So, we want to choose $\boldsymbol{v}_{h}$ such that

$$
\forall K \in \mathcal{T}_{h}, \quad \alpha \sum_{e \in \mathcal{E}_{h}, e \subset \partial K} \operatorname{meas}(e)\left(\boldsymbol{v}_{h} \cdot \boldsymbol{n}_{e}\right)\left(\boldsymbol{m}_{e}\right)=-\int_{K} q_{h} \psi_{K} \mathrm{~d} \boldsymbol{x} .
$$

This is achieved in the following way. Let $T_{h}$ be any tree associated with $\mathcal{T}_{h}$ (its existence follows from Lemma 5.1). We take the $\left(\boldsymbol{v}_{h} \cdot \boldsymbol{n}_{e}\right)\left(\boldsymbol{m}_{e}\right)$ equal to zero for all $e$ that do not intersect $T_{h}$. 
1) We start with the endpoints of this tree. Each endpoint is the barycenter of an element $K$ and the line crosses $\partial K$ inside one edge or face $e$. We thus take

$$
\left(\boldsymbol{v}_{h} \cdot \boldsymbol{n}_{e}\right)\left(\boldsymbol{m}_{e}\right)=-\frac{1}{\alpha \operatorname{meas}(e)} \int_{K} q_{h} \psi_{K} \mathrm{~d} \boldsymbol{x}
$$

2) We go back from this element along the tree. For any element $K$ but the one which contains the root, the part of the tree issued from the root intersects $\partial K$ in one edge or face $e$ and the part of the tree going to the endpoints intersects $\partial K$ in $k$ other edges $e_{i}, 1 \leq i \leq k$, with $1 \leq k \leq d$. Assuming by induction that the $\left(\boldsymbol{v}_{h} \cdot \boldsymbol{n}_{e_{i}}\right)\left(\boldsymbol{m}_{e_{i}}\right), 1 \leq i \leq k$, are known, we take

$$
\left(\boldsymbol{v}_{h} \cdot \boldsymbol{n}_{e}\right)\left(\boldsymbol{m}_{e}\right)=-\frac{1}{\operatorname{meas}(e)}\left(\frac{1}{\alpha} \int_{K} q_{h} \psi_{K} \mathrm{~d} \boldsymbol{x}+\sum_{i=1}^{k} \operatorname{meas}\left(e_{i}\right)\left(\boldsymbol{v}_{h} \cdot \boldsymbol{n}_{e_{i}}\right)\left(\boldsymbol{m}_{e_{i}}\right)\right) .
$$

3) For the element $K_{1}$ that contains the initial point, we know from the definition of $M_{h}^{N *}$ that

$$
\sum_{K \in \mathcal{T}_{h}} \int_{K} q_{h} \psi_{K} \mathrm{~d} \boldsymbol{x}=0
$$

So, since (5.5) now holds on all elements $K$ but $K_{1}$, summing it on all these elements, we obtain

$$
\begin{aligned}
\int_{K_{1}} q_{h} \psi_{K_{1}} \mathrm{~d} \boldsymbol{x} & =-\sum_{K \in \mathcal{T}_{h}, K \neq K_{1}} \int_{K} q_{h} \psi_{K} \mathrm{~d} \boldsymbol{x} \\
& =\sum_{K \in \mathcal{T}_{h}, K \neq K_{1}} \alpha \sum_{e \in \mathcal{E}_{h}, e \subset \partial K} \operatorname{meas}(e)\left(\boldsymbol{v}_{h} \cdot \boldsymbol{n}_{e}\right)\left(\boldsymbol{m}_{e}\right) \\
& =-\alpha \sum_{e \in \mathcal{E}_{h}, e \subset \partial K_{1}} \operatorname{meas}(e)\left(\boldsymbol{v}_{h} \cdot \boldsymbol{n}_{e}\right)\left(\boldsymbol{m}_{e}\right) .
\end{aligned}
$$

So this function $\boldsymbol{v}_{h}$ satisfies (5.3).

Now, we must bound the norm $\left\|\boldsymbol{v}_{h}\right\|_{H_{h}^{1}(\Omega)}$. We observe that, for each edge or face $e$ of an element of $\mathcal{T}_{h}$,

$$
\operatorname{meas}(e)\left|\left(\boldsymbol{v}_{h} \cdot \boldsymbol{n}_{e}\right)\left(\boldsymbol{m}_{e}\right)\right| \leq \frac{1}{\alpha} \sum_{K \in \mathcal{T}_{h}}\left|\int_{K} q_{h} \psi_{K} \mathrm{~d} \boldsymbol{x}\right| .
$$

Using twice the Cauchy-Schwarz inequality and the fact that $\left\|\psi_{K}\right\|_{L^{2}(K)}$ is equal to a constant times meas $(K)^{\frac{1}{2}}$, see (3.10), we obtain

$$
\left|\left(\boldsymbol{v}_{h} \cdot \boldsymbol{n}_{e}\right)\left(\boldsymbol{m}_{e}\right)\right| \leq c h_{e}^{1-d} \sum_{K \in \mathcal{T}_{h}}\left\|q_{h}\right\|_{L^{2}(K)} \operatorname{meas}(K)^{\frac{1}{2}} \leq c h_{e}^{1-d} \operatorname{meas}(\Omega)^{\frac{1}{2}}\left\|q_{h}\right\|_{L^{2}(\Omega)} .
$$

Combining this with (4.3) yields

$$
\left|\boldsymbol{v}_{h}\right|_{H_{h}^{1}(\Omega)} \leq c \operatorname{meas}(\Omega)^{\frac{1}{2}}\left\|q_{h}\right\|_{L^{2}(\Omega)}\left(\sum_{K \in \mathcal{T}_{h}} h_{K}^{-d}\right)^{\frac{1}{2}} \leq c\left(\inf _{K \in \mathcal{T}_{h}} h_{K}\right)^{-d} \operatorname{meas}(\Omega)\left\|q_{h}\right\|_{L^{2}(\Omega)} .
$$

Taking $r_{h}$ equal to $q_{h}$ in (5.3) and using this estimate leads to the desired result.

We now prove the inf-sup condition with $M_{h}^{N *}$ replaced by $M_{h}^{N 0}$. 
Lemma 5.3. There exists a constant $\beta_{N 0}$ independent of $h$ such that the following inf-sup condition holds

$$
\forall q_{h} \in M_{h}^{N 0}, \quad \sup _{\boldsymbol{v}_{h} \in X_{h}^{N}} \frac{b_{h}\left(\boldsymbol{v}_{h}, q_{h}\right)}{\left\|\boldsymbol{v}_{h}\right\|_{H_{h}^{1}(\Omega)}} \geq \frac{\beta_{N 0}}{\operatorname{meas}(\Omega)}\left(\inf _{K \in \mathcal{T}_{h}} h_{K}\right)^{d}\left\|q_{h}\right\|_{L^{2}(\Omega)} .
$$

Proof. Let us introduce an operator $L_{h}^{N}$ from $M_{h}^{N *}$ into $M_{h}^{N 0}$ defined by

$$
L_{h}^{N}\left(q_{h}^{*}\right)=q_{h}^{*}-\frac{\int_{\Omega} q_{h}^{*} \mathrm{~d} \boldsymbol{x}}{\int_{\Omega} \psi_{h} \mathrm{~d} \boldsymbol{x}} \psi_{h}
$$

Indeed, if $L_{h}^{N}\left(q_{h}^{*}\right)$ is zero, multiplying the previous formula by $\psi_{h}$ and integrating on $\Omega$ implies that

$$
\int_{\Omega} q_{h}^{*} \mathrm{~d} \boldsymbol{x}=0
$$

hence $q_{h}^{*}$ is zero and the operator $L_{h}^{N}$ is one-to-one. Moreover, since both $M_{h}^{N 0}$ and $M_{h}^{N *}$ have codimension 1 in $M_{h}^{N}$, it is an isomorphism from $M_{h}^{N *}$ onto $M_{h}^{N 0}$. Next, we derive from (3.10) that

$$
\int_{\Omega} \psi_{h} \mathrm{~d} \boldsymbol{x}=\frac{d !}{(2 d+1) !} \operatorname{meas}(\Omega), \quad \int_{\Omega} \psi_{h}^{2} \mathrm{~d} \boldsymbol{x}=\frac{2^{d+1} d !}{(3 d+2) !} \operatorname{meas}(\Omega),
$$

which, combined with the Cauchy-Schwarz inequality, yields the stability property

$$
\forall q_{h}^{*} \in M_{h}^{N *}, \quad\left\|L_{h}\left(q_{h}^{*}\right)\right\|_{L^{2}(\Omega)} \leq\left(1+\frac{2^{d+1}(2 d+1) !^{2}}{d !(3 d+2) !}\right)^{\frac{1}{2}}\left\|q_{h}^{*}\right\|_{L^{2}(\Omega)} .
$$

Let now $q_{h}$ be any function in $M_{h}^{0}$. The idea is to take $q_{h}^{*}=\left(L_{h}^{N}\right)^{-1}\left(q_{h}\right)$. Indeed, since $q_{h}-q_{h}^{*}$ is equal to a constant times $\psi_{h}$, we derive from Lemma 3.4 and from Lemma 5.2:

$$
\sup _{\boldsymbol{v}_{h} \in X_{h}^{N}} \frac{b_{h}\left(\boldsymbol{v}_{h}, q_{h}\right)}{\left\|\boldsymbol{v}_{h}\right\|_{H_{h}^{1}(\Omega)}}=\sup _{\boldsymbol{v}_{h} \in X_{h}^{N}} \frac{b_{h}\left(\boldsymbol{v}_{h}, q_{h}^{*}\right)}{\left\|\boldsymbol{v}_{h}\right\|_{H_{h}^{1}(\Omega)}} \geq \frac{\beta_{N *}}{\operatorname{meas}(\Omega)}\left(\inf _{K \in \mathcal{T}_{h}} h_{K}\right)^{d}\left\|q_{h}^{*}\right\|_{L^{2}(\Omega)},
$$

which combined with (5.7) gives the desired inf-sup condition.

Exactly as in Section 4, Lemma 5.2 (or Lem. 5.3) has a first and obvious consequence.

Corollary 5.4. The space of functions $q_{h}$ in $M_{h}^{N}$ such that

$$
\forall \boldsymbol{v}_{h} \in X_{h}^{N}, \quad b_{h}\left(\boldsymbol{v}_{h}, q_{h}\right)=0,
$$

has dimension 1 and is spanned by the function $\psi_{h}$.

Unfortunately, we are not able to prove the inf-sup condition between $X_{h}^{N}$ and $M_{h}^{N 0}$ with a constant independent of $h$. However, in a final step, we establish an optimal inf-sup condition between $X_{h}$ and $M_{h}^{N 0}$. Its proof relies on Fortin's trick [10], combined with the approximation properties of the operator $\Pi_{h}$ stated in Lemma 3.2 .

Lemma 5.5. There exists a constant $\beta_{N}$ independent of $h$ such that the following inf-sup condition holds

$$
\forall q_{h} \in M_{h}^{N 0}, \quad \sup _{\boldsymbol{v}_{h} \in X_{h}} \frac{b_{h}\left(\boldsymbol{v}_{h}, q_{h}\right)}{\left\|\boldsymbol{v}_{h}\right\|_{H_{h}^{1}(\Omega)}} \geq \beta_{N}\left\|q_{h}\right\|_{L^{2}(\Omega)} .
$$


Proof. Let $q_{h}$ be any function in $M_{h}^{N 0}$. It admits the expansion

$$
q_{h}=\sum_{K \in \mathcal{T}_{h}} \lambda_{K} \psi_{K}
$$

so that, thanks to (3.10),

$$
\left\|q_{h}\right\|_{L^{2}(\Omega)}^{2}=\frac{2^{d+1} d !}{(3 d+2) !} \sum_{K \in \mathcal{T}_{h}} \lambda_{K}^{2} \operatorname{meas}(K) .
$$

Since $q_{h}$ belongs to $L_{0}^{2}(\Omega)$, it follows from $(2.5)$ that there exists a function $\boldsymbol{v}$ in $H_{0}^{1}(\Omega)^{d}$ such that

$$
\operatorname{div} \boldsymbol{v}=-q_{h} \quad \text { and } \quad|\boldsymbol{v}|_{H^{1}(\Omega)^{d}} \leq \frac{1}{\beta}\left\|q_{h}\right\|_{L^{2}(\Omega)} .
$$

The idea is now to take $\boldsymbol{v}_{h}=\Pi_{h} \boldsymbol{v}$, for the operator $\Pi_{h}$ defined by (3.4). Indeed, with this choice, since $q_{h}$ vanishes on all $\partial K$, integrating by parts and using once more (3.10) yield

$$
b_{h}\left(\boldsymbol{v}_{h}, q_{h}\right)=-\sum_{K \in \mathcal{T}_{h}} \lambda_{K}\left(\operatorname{div} \boldsymbol{v}_{h}\right)_{\mid K} \int_{K} \psi_{K} \mathrm{~d} \boldsymbol{x}=-\frac{d !}{(2 d+1) !} \sum_{K \in \mathcal{T}_{h}} \lambda_{K} \operatorname{meas}(K)\left(\operatorname{div} \boldsymbol{v}_{h}\right)_{\mid K} .
$$

We also have

$$
\operatorname{meas}(K)\left(\operatorname{div} \boldsymbol{v}_{h}\right)_{\mid K}=\int_{K} \operatorname{div} \boldsymbol{v}_{h} \mathrm{~d} \boldsymbol{x}=\sum_{e \subset \partial K} \int_{e} \boldsymbol{v}_{h} \cdot \boldsymbol{n}_{e} \mathrm{~d} \tau .
$$

Thanks to the definition of $\Pi_{h}$, see (3.4), this yields

$$
\operatorname{meas}(K)\left(\operatorname{div} \boldsymbol{v}_{h}\right)_{\mid K}=\sum_{e \subset \partial K} \int_{e} \boldsymbol{v} \cdot \boldsymbol{n}_{e} \mathrm{~d} \tau=\int_{K} \operatorname{div} \boldsymbol{v} \mathrm{d} \boldsymbol{x}
$$

so that

$$
b_{h}\left(\boldsymbol{v}_{h}, q_{h}\right)=\frac{d !}{(2 d+1) !} \sum_{K \in \mathcal{T}_{h}} \lambda_{K}^{2} \int \psi_{K} \mathrm{~d} \boldsymbol{x}=\left(\frac{d !}{(2 d+1) !}\right)^{2} \sum_{K \in \mathcal{T}_{h}} \lambda_{K}^{2} \operatorname{meas}(K)=c\left\|q_{h}\right\|_{L^{2}(\Omega)}^{2} .
$$

Moreover, from (3.5), we obtain

$$
\left\|\boldsymbol{v}_{h}\right\|_{H_{h}^{1}(\Omega)} \leq c|\boldsymbol{v}|_{H^{1}(\Omega)^{d}} \leq \frac{c}{\beta}\left\|q_{h}\right\|_{L^{2}(\Omega)},
$$

which gives the desired condition.

Remark. Let us consider once more the union $\bar{\Omega}_{\boldsymbol{a}}$ of all elements $K$ in $\mathcal{T}_{h}$ that contain a fixed vertex $\boldsymbol{a}$ of an element in $\mathcal{T}_{h}$. The same arguments as for Lemmas 5.2 and 5.3 lead to an optimal inf-sup condition between the space $X_{\boldsymbol{a}}^{N}$ of restrictions to $\Omega_{\boldsymbol{a}}$ of functions in $X_{h}^{N}$ which vanish in the midpoints of the edges or barycenters of the faces contained in $\partial \Omega_{\boldsymbol{a}}$ and the space $M_{\boldsymbol{a}}^{N 0}=M_{\boldsymbol{a}}^{N} \cap L_{0}^{2}\left(\Omega_{\boldsymbol{a}}\right)$, where $M_{\boldsymbol{a}}^{N}$ denote the space of restrictions of functions in $M_{h}^{N}$ to $\Omega_{a}$. Next, combining a modified version of Lemma 5.6 with the Boland and Nicolaides argument [4] yields an optimal inf-sup condition as (5.9), but with $X_{h}$ replaced by the sum of $X_{h}^{N}$ and a smaller subspace of $X_{h}^{T}$.

Remark. Let $P_{h}^{N}$ denote the operator which, with each $\boldsymbol{v}_{h}$ in $X_{h}$, associates the only element $P_{h}^{N} \boldsymbol{v}_{h}$ of $X_{h}^{N}$ such that $\boldsymbol{v}_{h}-P_{h}^{N} \boldsymbol{v}_{h}$ belongs to $X_{h}^{T}$, and let $\mu_{h}$ stands for its norm (the spaces $X_{h}$ and $X_{h}^{N}$ being provided 
with the norm $\left.\|\cdot\|_{H_{h}^{1}(\Omega)}\right)$. Then, we derive from Lemmas 3.3 and 5.5 the inf-sup condition

$$
\forall q_{h} \in M_{h}^{N 0}, \quad \sup _{\boldsymbol{v}_{h} \in X_{h}^{N}} \frac{b_{h}\left(\boldsymbol{v}_{h}, q_{h}\right)}{\left\|\boldsymbol{v}_{h}\right\|_{H_{h}^{1}(\Omega)}} \geq \frac{\beta_{N}}{\mu_{h}}\left\|q_{h}\right\|_{L^{2}(\Omega)} .
$$

However, it follows from (3.15) that $\mu_{h}$ is not bounded independently of $h$.

\section{The Global InF-SUP CONDition AND ITS CONSEQUENCES}

Thanks to the previous results, we are now in a position to establish the inf-sup condition between the spaces $X_{h}$ and $M_{h}^{0}$, however with a constant depending on $h$. Its proof relies in fact on a modal analogue of the Boland and Nicolaides argument [4]. For any $q_{h}$ in $M_{h}$, we agree to denote by $\left(q_{h}^{T}, q_{h}^{N}\right)$ the only pair in $M_{h}^{T} \times M_{h}^{N}$ such that $q_{h}=q_{h}^{T}+q_{h}^{N}$.

Theorem 6.1. If Hypothesis 4.1 holds, there exists a constant $\tilde{\beta}$ independent of $h$ such that the following inf-sup condition holds

$$
\forall q_{h} \in M_{h}^{0}, \quad \sup _{\boldsymbol{v}_{h} \in X_{h}} \frac{b_{h}\left(\boldsymbol{v}_{h}, q_{h}\right)}{\left\|\boldsymbol{v}_{h}\right\|_{H_{h}^{1}(\Omega)}} \geq \tilde{\beta}\left(\inf _{K \in \mathcal{T}_{h}} h_{K}\right)\left\|q_{h}\right\|_{L^{2}(\Omega)}
$$

Proof. Let $q_{h}$ be any function in $M_{h}^{0}$. Setting for a while $h_{-}=\inf _{K \in \mathcal{T}_{h}} h_{K}$, we know from (4.1) that there exists a function $\boldsymbol{v}_{h}^{T}$ in $X_{h}^{T}$ such that

$$
b_{h}\left(\boldsymbol{v}_{h}^{T}, q_{h}^{T}\right)=h_{-}\left\|q_{h}^{T}\right\|_{L^{2}(\Omega)}^{2} \quad \text { and } \quad\left\|\boldsymbol{v}_{h}^{T}\right\|_{H_{h}^{1}(\Omega)} \leq \frac{1}{\beta_{T 0}}\left\|q_{h}^{T}\right\|_{L^{2}(\Omega)},
$$

and similarly, from (5.9), we know that there exists a function $\boldsymbol{w}_{h}$ in $X_{h}$ such that

$$
b_{h}\left(\boldsymbol{w}_{h}, q_{h}^{N}\right)=\left\|q_{h}^{N}\right\|_{L^{2}(\Omega)}^{2} \quad \text { and } \quad\left\|\boldsymbol{w}_{h}\right\|_{H_{h}^{1}(\Omega)} \leq \frac{1}{\beta_{N}}\left\|q_{h}^{N}\right\|_{L^{2}(\Omega)} .
$$

The idea is now to take $\boldsymbol{v}_{h}=\boldsymbol{v}_{h}^{T}+\mu \boldsymbol{w}_{h}$ for a positive constant $\mu$. Indeed, using (6.2), (6.3) and the orthogonality property (3.9), we have

$$
b_{h}\left(\boldsymbol{v}_{h}, q_{h}\right)=h_{-}\left\|q_{h}^{T}\right\|_{L^{2}(\Omega)}^{2}+\mu\left\|q_{h}^{N}\right\|_{L^{2}(\Omega)}^{2}+\mu b_{h}\left(\boldsymbol{w}_{h}, q_{h}^{T}\right)
$$

Using the continuity property (3.1) and once more (6.3), we derive

$$
b_{h}\left(\boldsymbol{v}_{h}, q_{h}\right) \geq h_{-}\left\|q_{h}^{T}\right\|_{L^{2}(\Omega)}^{2}+\mu\left\|q_{h}^{N}\right\|_{L^{2}(\Omega)}^{2}-\frac{c}{\beta_{N}} \mu\left\|q_{h}^{T}\right\|_{L^{2}(\Omega)}\left\|q_{h}^{N}\right\|_{L^{2}(\Omega)},
$$

whence

$$
b_{h}\left(\boldsymbol{v}_{h}, q_{h}\right) \geq \frac{h_{-}}{2}\left\|q_{h}^{T}\right\|_{L^{2}(\Omega)}^{2}+\mu\left(1-\frac{c^{2} \mu}{2 \beta_{N}^{2} h_{-}}\right)\left\|q_{h}^{N}\right\|_{L^{2}(\Omega)}^{2} .
$$

Taking $\mu=\frac{\beta_{N}^{2} h_{-}}{c^{2}}$ yields

$$
b_{h}\left(\boldsymbol{v}_{h}, q_{h}\right) \geq \inf \left\{\frac{h_{-}}{2}, \frac{\beta_{N}^{2} h_{-}}{2 c^{2}}\right\}\left(\left\|q_{h}^{T}\right\|_{L^{2}(\Omega)}^{2}+\left\|q_{h}^{N}\right\|_{L^{2}(\Omega)}^{2}\right) .
$$


On the other hand, using (6.2) and (6.3) yields

$$
\begin{aligned}
\left|\boldsymbol{v}_{h}\right|_{H_{h}^{1}(\Omega)} \leq\left|\boldsymbol{v}_{h}^{T}\right|_{H_{h}^{1}(\Omega)}+\mu\left|\boldsymbol{w}_{h}\right|_{H_{h}^{1}(\Omega)} & \leq \frac{1}{\beta_{T 0}}\left\|q_{h}^{T}\right\|_{L^{2}(\Omega)}+\frac{\mu}{\beta_{N}}\left\|q_{h}^{N}\right\|_{L^{2}(\Omega)} \\
& \leq\left(\frac{1}{\beta_{T 0}^{2}}+\frac{\mu^{2}}{\beta_{N}^{2}}\right)^{\frac{1}{2}}\left(\left\|q_{h}^{T}\right\|_{L^{2}(\Omega)}^{2}+\left\|q_{h}^{N}\right\|_{L^{2}(\Omega)}^{2}\right)^{\frac{1}{2}} .
\end{aligned}
$$

Combining the previous properties together with the triangular inequality

$$
\left\|q_{h}\right\|_{L^{2}(\Omega)} \leq\left\|q_{h}^{T}\right\|_{L^{2}(\Omega)}+\left\|q_{h}^{N}\right\|_{L^{2}(\Omega)} \leq \sqrt{2}\left(\left\|q_{h}^{T}\right\|_{L^{2}(\Omega)}^{2}+\left\|q_{h}^{N}\right\|_{L^{2}(\Omega)}^{2}\right)^{\frac{1}{2}},
$$

leads to the desired inf-sup condition.

The constant in the inf-sup condition (6.1) is not independent of $h$. Even if counter-examples are rather difficult to exhibit, numerical experiments confirm this lack of optimality of the discretization. Moreover,

- as explained in Section 4, improving the inf-sup condition between $X_{h}^{T}$ and $M_{h}^{T 0}$ does not seem possible,

- if all the degrees of freedom of the space $X_{h}$ are needed for proving optimal inf-sup conditions for both the subspaces $M_{h}^{T 0}$ and $M_{h}^{N 0}$, the fact that the angle between $M_{h}^{T}$ and $M_{h}^{N}$ is bounded from below independently of $h$ (see the final remark of Section 3) makes the optimal global inf-sup condition unlikely.

So, the main consequence of Theorem 6.1 is the well-posedness of Problem (2.6).

Corollary 6.2. If Hypothesis 4.1 holds, for any data $\boldsymbol{f}$ in $L^{2}(\Omega)^{d}$, problem (2.6) has a unique solution $\left(\boldsymbol{u}_{h}, p_{h}\right)$ in $X_{h} \times M_{h}^{0}$. Moreover, this solution satisfies

$$
\left\|\boldsymbol{u}_{h}\right\|_{H_{h}^{1}(\Omega)}+\left(\inf _{K \in \mathcal{T}_{h}} h_{K}\right)\left\|p_{h}\right\|_{L^{2}(\Omega)} \leq c\|\boldsymbol{f}\|_{L^{2}(\Omega)^{d}},
$$

for a constant $c$ independent of $h$.

As proven in [11, Chap. II, Th. 1.1], the second consequence of this condition is that the following version of Strang's lemma holds in this case: if $\beta_{h}$ stands for the constant $\tilde{\beta}\left(\inf _{K \in \mathcal{T}_{h}} h_{K}\right)$ of (6.1),

$$
\begin{aligned}
\left\|\boldsymbol{u}-\boldsymbol{u}_{h}\right\|_{H_{h}^{1}(\Omega)}+\beta_{h}\left\|p-p_{h}\right\|_{L^{2}(\Omega)} \leq & c\left(\inf _{\boldsymbol{v}_{h} \in V_{h}}\left\|\boldsymbol{u}-\boldsymbol{v}_{h}\right\|_{H_{h}^{1}(\Omega)}+\inf _{q_{h} \in M_{h}^{0}}\left\|p-q_{h}\right\|_{L^{2}(\Omega)}\right. \\
& \left.+\sup _{\boldsymbol{w}_{h} \in X_{h}} \frac{\sum_{e \in \mathcal{E}_{h}} \int_{e} \partial_{n_{e}} \boldsymbol{u} \cdot\left[\boldsymbol{w}_{h}\right] \mathrm{d} \tau}{\left\|\boldsymbol{w}_{h}\right\|_{H_{h}^{1}(\Omega)}}\right)
\end{aligned}
$$

where the constant $c$ is independent of $h$ and the notation [.] for the jump through an edge or a face inside $\Omega$ is extended to edges or faces contained in $\partial \Omega$ to denote the trace. The first two terms in the right-hand side represent the approximation error, while the last one represents the consistency error. This consistency term can be evaluated in a standard way, by noting that each jump $\left[\boldsymbol{w}_{h}\right]$ is orthogonal to the constants in $L^{2}(e)$.

We also recall the estimate which still relies on condition (6.1), see [11, Chap. II, (1.16)]

$$
\inf _{\boldsymbol{v}_{h} \in V_{h}}\left\|\boldsymbol{u}-\boldsymbol{v}_{h}\right\|_{H_{h}^{1}(\Omega)} \leq c\left(1+\beta_{h}^{-1}\right) \inf _{\boldsymbol{w}_{h} \in X_{h}}\left\|\boldsymbol{u}-\boldsymbol{w}_{h}\right\|_{H_{h}^{1}(\Omega)} .
$$

But this last estimate is unsufficient to prove the convergence of the discretization, even for the velocity. So we must evaluate the distance of $\boldsymbol{u}$ to $V_{h}$ "by hand".

Proposition 6.3. There exists a constant $c$ independent of $h$ such that the following estimate holds for all functions $\boldsymbol{u}$ in $V \cap H^{s+1}(\Omega)^{d}, 0<s \leq 1$ :

$$
\inf _{\boldsymbol{v}_{h} \in V_{h}}\left\|\boldsymbol{u}-\boldsymbol{v}_{h}\right\|_{H_{h}^{1}(\Omega)} \leq c h^{s}\|\boldsymbol{u}\|_{H^{s+1}(\Omega)^{d}}
$$


Proof. It is performed in two steps.

1) By integration by parts, it follows from (3.10) that, for all $K$ in $\mathcal{T}_{h}$,

$$
\begin{aligned}
b_{h}\left(\Pi_{h} \boldsymbol{u}, \psi_{K}\right) & =-\left(\operatorname{div} \Pi_{h} \boldsymbol{u}\right)_{\mid K} \int_{K} \psi_{K} \mathrm{~d} \boldsymbol{x} \\
& =-\frac{d !}{(2 d+1) !} \int_{K} \operatorname{div} \Pi_{h} \boldsymbol{u} \mathrm{d} \boldsymbol{x}=-\frac{d !}{(2 d+1) !} \sum_{e \subset \partial K} \int_{e} \Pi_{h} \boldsymbol{u} \cdot \boldsymbol{n}_{e} \mathrm{~d} \boldsymbol{x} .
\end{aligned}
$$

Then using the definition $(3.4)$ of $\Pi_{h}$ yields

$$
b_{h}\left(\Pi_{h} \boldsymbol{u}, \psi_{K}\right)=-\frac{d !}{(2 d+1) !} \sum_{e \subset \partial K} \int_{e} \boldsymbol{u} \cdot \boldsymbol{n}_{e} \mathrm{~d} \boldsymbol{x}=-\frac{d !}{(2 d+1) !} \int_{K} \operatorname{div} \boldsymbol{u} \mathrm{d} \boldsymbol{x}=0
$$

So, we derive

$$
\forall q_{h}^{N} \in M_{h}^{N 0}, \quad b_{h}\left(\Pi_{h} \boldsymbol{u}, q_{h}^{N}\right)=0 .
$$

Moreover, since $\Pi_{h}$ leaves invariant all functions of $X_{h}$, combining the approximation properties of the conforming space $X_{h} \cap H_{0}^{1}(\Omega)^{d}$ with (3.5) leads to

$$
\left\|\boldsymbol{u}-\Pi_{h} \boldsymbol{u}\right\|_{H_{h}^{1}(\Omega)} \leq c h^{s}\|\boldsymbol{u}\|_{H^{s+1}(\Omega)^{d}} .
$$

2) The idea is now to take $\boldsymbol{v}_{h}=\Pi_{h} \boldsymbol{u}+\boldsymbol{v}_{h}^{T}$, where $\boldsymbol{v}_{h}^{T}$ is a function in $X_{h}^{T}$ satisfying

$$
\forall q_{h}^{T} \in M_{h}^{T 0}, \quad b_{h}\left(\boldsymbol{v}_{h}^{T}, q_{h}^{T}\right)=-b_{h}\left(\Pi_{h} \boldsymbol{u}, q_{h}^{T}\right) .
$$

Indeed, thanks to (6.8), (6.10) and the orthogonality property (3.9), the corresponding function $\boldsymbol{v}_{h}$ belongs to $V_{h}$. Also, from the inf-sup condition (4.1), system (6.10) has a unique solution $\boldsymbol{v}_{h}^{T}$ in the orthogonal of $V_{h} \cap X_{h}^{T}$ in $X_{h}^{T}$. Moreover, since $\boldsymbol{u}$ belongs to $V$, we have

$$
-b_{h}\left(\Pi_{h} \boldsymbol{u}, q_{h}^{T}\right)=b_{h}\left(\boldsymbol{u}-\Pi_{h} \boldsymbol{u}, q_{h}^{T}\right) \leq \sum_{K \in \mathcal{T}_{h}}\left\|\boldsymbol{u}-\Pi_{h} \boldsymbol{u}\right\|_{L^{2}(K)}\left|q_{h}^{T}\right|_{H^{1}(K)}
$$

So, due to the identity

$$
\boldsymbol{u}-\Pi_{h} \boldsymbol{u}=\left(\mathrm{Id}-\Pi_{h}\right)\left(\boldsymbol{u}-\Pi_{h} \boldsymbol{u}\right),
$$

applying (3.6) and a Cauchy-Schwarz inequality yields, for the norm $\|\cdot\|_{L_{h}^{2}(\Omega)}$ defined in (4.6),

$$
-b_{h}\left(\Pi_{h} \boldsymbol{u}, q_{h}^{T}\right) \leq c\left\|\boldsymbol{u}-\Pi_{h} \boldsymbol{u}\right\|_{H_{h}^{1}(\Omega)}\left\|q_{h}^{T}\right\|_{L_{h}^{2}(\Omega)} .
$$

So it follows from the inf-sup condition (4.5) (see [11, Chap. I, Lem. 4.1]) that the solution $\boldsymbol{v}_{h}^{T}$ of (6.10) satisfies

$$
\left\|\boldsymbol{v}_{h}^{T}\right\|_{H_{h}^{1}(\Omega)} \leq \frac{c}{\beta_{T 0}}\left\|\boldsymbol{u}-\Pi_{h} \boldsymbol{u}\right\|_{H_{h}^{1}(\Omega)}
$$

The desired estimate follows by inserting (6.9) and (6.11) in the triangular inequality

$$
\left\|\boldsymbol{u}-\boldsymbol{v}_{h}\right\|_{H_{h}^{1}(\Omega)} \leq\left\|\boldsymbol{u}-\Pi_{h} \boldsymbol{u}\right\|_{H_{h}^{1}(\Omega)}+\left\|\boldsymbol{v}_{h}^{T}\right\|_{H_{h}^{1}(\Omega)} .
$$

Combining Proposition 6.3 with (6.5) leads to the first estimate on the velocity. 
Theorem 6.4. If Hypothesis 4.1 holds, for any data $\boldsymbol{f}$ in $L^{2}(\Omega)^{d}$ and if, moreover, the solution (u,p) of Problem (2.1) belongs to the space $H^{s+1}(\Omega)^{d} \times H^{s}(\Omega)$ for a real number $s, 0<s \leq 1$, there exists a constant $c$ independent of $h$ such that the following error estimate holds between the velocity $\boldsymbol{u}$ of Problem (2.1) and the velocity $\boldsymbol{u}_{h}$ of Problem (2.6):

$$
\left\|\boldsymbol{u}-\boldsymbol{u}_{h}\right\|_{H_{h}^{1}(\Omega)^{d}} \leq c h^{s}\left(\|\boldsymbol{u}\|_{H^{s+1}(\Omega)^{d}}+\|p\|_{H^{s}(\Omega)}\right)
$$

Proof. Estimating the first term in the right-hand side of (6.5) is performed in Proposition 6.3 and estimating the second term follows from the standard approximation properties of the space $M_{h}^{T 0}$. As already hinted, the last term is evaluated thanks to the next inequality that holds for any constant $\boldsymbol{c}_{e}$

$$
\int_{e} \partial_{n_{e}} \boldsymbol{u} \cdot\left[\boldsymbol{w}_{h}\right] \mathrm{d} \tau=\int_{e}\left(\partial_{n_{e}} \boldsymbol{u}-\boldsymbol{c}_{e}\right) \cdot\left[\boldsymbol{w}_{h}\right] \mathrm{d} \tau \leq\left\|\partial_{n_{e}} \boldsymbol{u}-\boldsymbol{c}_{e}\right\|_{H^{-\frac{1}{2}(e)}}\left\|\left[\boldsymbol{w}_{h}\right]\right\|_{H^{\frac{1}{2}}(e)}
$$

So taking $\boldsymbol{c}_{e}$ equal to the mean value of $\partial_{n_{e}} \boldsymbol{u}$ on $e$ and going to a reference element as in the proof of Lemma 3.1 lead to, for any $\boldsymbol{w}_{h}$ in $X_{h}$,

$$
\frac{\sum_{e \in \mathcal{E}_{h}} \int_{e} \partial_{n_{e}} \boldsymbol{u} \cdot\left[\boldsymbol{w}_{h}\right] \mathrm{d} \tau}{\left\|\boldsymbol{w}_{h}\right\|_{H_{h}^{1}(\Omega)}} \leq c h^{s}\|\boldsymbol{u}\|_{H^{s+1}(\Omega)^{d}} .
$$

This concludes the proof.

We conclude by an error estimate for the velocity in $L^{2}(\Omega)^{d}$ which relies on rather standard arguments.

Proposition 6.5. If the assumptions of Theorem 6.4 hold, there exist a parameter $\alpha, \frac{1}{2}<\alpha \leq 1$, only depending on the geometry of $\Omega$, and a constant $c$ independent of $h$ such that the following error estimate holds between the velocity $\boldsymbol{u}$ of problem (2.1) and the velocity $\boldsymbol{u}_{h}$ of problem (2.6):

$$
\left\|\boldsymbol{u}-\boldsymbol{u}_{h}\right\|_{L^{2}(\Omega)^{d}} \leq c h^{s+\alpha}\left(\|\boldsymbol{u}\|_{H^{s+1}(\Omega)^{d}}+\|p\|_{H^{s}(\Omega)}\right) .
$$

If the domain $\Omega$ is convex, $\alpha$ is equal to 1.

Proof. We start from the formula

$$
\left\|\boldsymbol{u}-\boldsymbol{u}_{h}\right\|_{L^{2}(\Omega)^{d}}=\sup _{\boldsymbol{g} \in L^{2}(\Omega)^{d}} \frac{\int_{\Omega}\left(\boldsymbol{u}-\boldsymbol{u}_{h}\right)(\boldsymbol{x}) \cdot \boldsymbol{g}(\boldsymbol{x}) \mathrm{d} \boldsymbol{x}}{\|\boldsymbol{g}\|_{L^{2}(\Omega)^{d}}}
$$

and with any $\boldsymbol{g}$ in $L^{2}(\Omega)^{d}$, we associate the solution $(\boldsymbol{w}, r)$ of the Stokes problem

$$
\begin{cases}-\Delta \boldsymbol{w}+\operatorname{grad} r=\boldsymbol{g} & \text { in } \Omega, \\ \operatorname{div} \boldsymbol{w}=0 & \text { in } \Omega, \\ \boldsymbol{w}=\mathbf{0} & \text { on } \partial \Omega .\end{cases}
$$

We recall that there exists an $\alpha, \frac{1}{2}<\alpha \leq 1$ (equal to 1 when $\Omega$ is convex) such that this solution belongs to $H^{\alpha+1}(\Omega)^{d} \times H^{\alpha}(\Omega)$ and satisfies

$$
\|\boldsymbol{w}\|_{H^{\alpha+1}(\Omega)^{d}}+\|r\|_{H^{\alpha}(\Omega)} \leq c\|\boldsymbol{g}\|_{L^{2}(\Omega)^{d}} .
$$


Next, we obtain, for any $\boldsymbol{w}_{h}$ in $V_{h}$ and $r_{h}$ in $M_{h}$,

$$
\begin{aligned}
\int_{\Omega}\left(\boldsymbol{u}-\boldsymbol{u}_{h}\right)(\boldsymbol{x}) \cdot \boldsymbol{g}(\boldsymbol{x}) \mathrm{d} \boldsymbol{x}= & a_{h}\left(\boldsymbol{u}-\boldsymbol{u}_{h}, \boldsymbol{w}-\boldsymbol{w}_{h}\right)+b_{h}\left(\boldsymbol{u}-\boldsymbol{u}_{h}, r-r_{h}\right) \\
& +\sum_{e \in \mathcal{E}_{h}} \int_{e} \int_{e} \partial_{n_{e}} \boldsymbol{w} \cdot\left[\boldsymbol{u}_{h}\right] \mathrm{d} \tau+\sum_{e \in \mathcal{E}_{h}} \int_{e} \int_{e} \partial_{n_{e}} \boldsymbol{u} \cdot\left[\boldsymbol{w}_{h}\right] \mathrm{d} \tau
\end{aligned}
$$

Thanks to (6.16), estimating the first terms relies on the approximation properties of $V_{h}$, see Proposition 6.3 , and of $M_{h}$, while estimating the two last ones relies on the analogue of (6.13). Inserting this in (6.15) leads to the desired estimate.

Remark. If the assumptions of Theorem 6.4 hold, the only estimate on the discrete pressure that can be deduced from the previous line writes

$$
\left\|p_{h}\right\|_{L^{2}(\Omega)} \leq c h^{s}\left(\inf _{K \in \mathcal{T}_{h}} h_{K}\right)^{-1}\left(\|\boldsymbol{u}\|_{H^{s+1}(\Omega)^{d}}+\|p\|_{H^{s}(\Omega)}\right)
$$

So, at least when $\Omega$ is convex and the family of triangulations $\left(\mathcal{T}_{h}\right)_{h}$ is uniformly regular (in the sense that the ratio $\left(\inf _{K \in \mathcal{T}_{h}} h_{K}\right) / h$ is larger than a constant independent of $h$ ), the norm $\left\|p_{h}\right\|_{L^{2}(\Omega)}$ is bounded independently of $h$.

To overcome this lack of convergence of the discrete pressure, let us finally consider the "reduced" problem: find $\left(\boldsymbol{u}_{h}, p_{h}\right)$ in $X_{h} \times M_{h}^{T 0}$ such that

$$
\begin{array}{ll}
\forall \boldsymbol{v}_{h} \in X_{h}, & a_{h}\left(\boldsymbol{u}_{h}, \boldsymbol{v}_{h}\right)+b_{h}\left(\boldsymbol{v}_{h}, p_{h}\right)=\int_{\Omega} \boldsymbol{f}(\boldsymbol{x}) \cdot \boldsymbol{v}_{h}(\boldsymbol{x}) \mathrm{d} \boldsymbol{x}, \\
\forall q_{h} \in M_{h}^{T 0}, & b_{h}\left(\boldsymbol{u}_{h}, q_{h}\right)=0 .
\end{array}
$$

Indeed, it follows from Proposition 4.5 that, if Hypothesis 4.1 holds, an optimal inf-sup condition holds between $X_{h}$ and $M_{h}^{T 0}$. This condition and the standard approximation properties of the spaces $X_{h}$ (which contains the "conforming" space of piecewise affine continuous functions vanishing on $\partial \Omega$ ) and $M_{h}^{T}$, combined with the analogues of (6.5) and (6.6) (but with $\beta_{h}$ replaced by $\beta_{T}$ ), lead to the following result.

Proposition 6.6. If Hypothesis 4.1 holds, for any data $\boldsymbol{f}$ in $L^{2}(\Omega)^{d}$, problem (6.19) has a unique solution $\left(\boldsymbol{u}_{h}, p_{h}\right)$ in $X_{h} \times M_{h}^{T 0}$. Moreover, this solution satisfies

$$
\left\|\boldsymbol{u}_{h}\right\|_{H_{h}^{1}(\Omega)}+\left\|p_{h}\right\|_{L^{2}(\Omega)} \leq c\|\boldsymbol{f}\|_{L^{2}(\Omega)^{d}}
$$

for a constant $c$ independent of $h$. If moreover, the solution $(\boldsymbol{u}, p)$ of Problem (2.1) belongs to the space $H^{s+1}(\Omega)^{d} \times H^{s}(\Omega)$ for a real number $s, 0<s \leq 1$, there exists a constant $c$ independent of $h$ such that the following error estimate holds between this solution and the solution $\left(\boldsymbol{u}_{h}, p_{h}\right)$ of Problem (6.19):

$$
\left\|\boldsymbol{u}-\boldsymbol{u}_{h}\right\|_{H_{h}^{1}(\Omega)}+\left\|p-p_{h}\right\|_{L^{2}(\Omega)} \leq c h^{s}\left(\|\boldsymbol{u}\|_{H^{s+1}(\Omega)^{d}}+\|p\|_{H^{s}(\Omega)}\right)
$$

Let us finally consider Problem (6.19) with $M_{h}^{T 0}$ replaced by $M_{h}^{N 0}$. From Lemma 5.5, this problem has a unique solution which still satisfies (6.20). However the approximation properties of the space $M_{h}^{N 0}$ are unsufficient for estimate (6.21) to be fully valid in this case, only the estimate concerning the velocity holds.

\section{An Algorithm FOR SOlving the Discrete PROBlem}

Problem (2.6) is equivalent to the square linear system

$$
\left(\begin{array}{cc}
A & B \\
B^{T} & 0
\end{array}\right)\left(\begin{array}{l}
U \\
P
\end{array}\right)=\left(\begin{array}{c}
F \\
0
\end{array}\right)
$$


where the vector $U$ is made of the values of $\boldsymbol{u}_{h}$ at the midpoints of all edges or barycenters of all faces $e$ that are not contained in $\partial \Omega$ and the vector $P$ is made of the values of $p_{h}$ at all vertices and barycenters of the elements $K$ of $\mathcal{T}_{h}$ (the two further orthogonality conditions in $M_{h}^{0}$ are usually enforced in a further step). Since the matrix $A$ is symmetric and positive definite, system (7.1) is also symmetric, however its size is very large, equal to $\operatorname{dim} X_{h}+\operatorname{dim} M_{h}$.

So the key idea for solving system (7.1) consists in separating the unknowns $U$ and $P$. For instance, the well-known Uzawa's algorithm [1] consists in eliminating the quantity $U$ from the first line in (7.1), leading to the system of two uncoupled equations

$$
B^{T} A^{-1} B P=B^{T} A^{-1} F, \quad A U=F-B P .
$$

The matrices in the right-hand member of these two equations are still symmetric, so that they can be solved by the conjugate gradient algorithm. However the first equation requires to invert the large matrix $A$. Moreover this algorithm is more efficient when the discrete space of pressures has a small size, which is not the case for the element considered in this paper.

Another idea, developed by Hecht [12] for the Crouzeix-Raviart element, relies on the reduced discrete problem (2.11). Indeed, for the space $V_{h}$ defined in (2.10), we introduce a fixed space $W_{h}$ such that

$$
X_{h}=V_{h} \oplus W_{h} .
$$

Note that, since $M_{h}^{0}$ contains no spurious modes,

$$
K_{h}=\operatorname{dim} V_{h}=\operatorname{dim} X_{h}-\operatorname{dim} M_{h}^{0}, \quad L_{h}=\operatorname{dim} W_{h}=\operatorname{dim} M_{h}^{0} .
$$

Next, we assume that a basis $\left\{v_{1}, \ldots, v_{K_{h}}\right\}$ of $V_{h}$ and a basis $\left\{w_{1}, \ldots, w_{L_{h}}\right\}$ of $W_{h}$ are known and we denote by $U_{1}$ the vector of the coefficients of $\boldsymbol{u}_{h}$ in $\left\{v_{1}, \ldots, v_{K_{h}}\right\}$. Using Problem (2.11), we observe that (7.1) is equivalent to the system of two uncoupled equations

$$
A_{1} U_{1}=F_{1}, \quad B_{2} P=F_{2}-A_{2} U_{1} .
$$

The matrix $A_{1}$ is still symmetric and positive definite, made of the $a_{h}\left(v_{i}, v_{j}\right), 1 \leq i, j \leq K_{h}$. The matrix $B_{2}$ is made of the $b_{h}\left(w_{j}, p\right), 1 \leq j \leq L_{h}$, where $p$ runs through a basis of $M_{h}$, and becomes square when adding the two lines corresponding to the further orthogonality conditions in the definition of $M_{h}^{0}$.

So it remains to construct a basis of $V_{h}$ and $W_{h}$. Note moreover that the matrix $A_{2}$ and $B_{2}$ are sparse if those bases are made of functions with a local support. From now on and for simplicity, we assume that the domain $\Omega$ is two-dimensional and simply-connected. We refer to [12] for the extension to the case of multiply-connected domains where a finite number (independent of $h$ ) of further basis functions appear in $V_{h}$.

In a first step, we construct a basis of $V_{h}^{T}=V_{h} \cap X_{h}^{T}$. With each edge $e$ in $\mathcal{E}_{h}$, we associate the Lagrange function $\mu_{e}$, already introduced in the proof of Lemma 4.2, which belongs to $\mathcal{P}_{1}(K)$ for all $K$ in $\mathcal{T}_{h}$ and vanishes in all the midpoints of the edges of elements $K$ in $\mathcal{T}_{h}$ but in $\boldsymbol{m}_{e}$ where it is equal to 1 . When the edge $e$ is not contained in $\partial \Omega$, the support of $\mu_{e}$ is made of two elements $K$ of $\mathcal{T}_{h}$. Thus, since $\mu_{e}$ is nonnegative on these elements, we introduce the function $\tilde{\mu}_{e}$ equal to a constant times $\mu_{e}$, such that

$$
\int_{\Omega} \tilde{\mu}_{e} \mathrm{~d} \boldsymbol{x}=\operatorname{meas}(e) .
$$

Moreover, with any vertex $\boldsymbol{a}$ of a triangle in $\mathcal{T}_{h}$, we associate the standard Lagrange function $\varphi_{\boldsymbol{a}}$ in $M_{h}^{T}$, equal to 1 in $\boldsymbol{a}$ and to 0 in all other vertices.

Finally, we denote by $\mathcal{T}_{h}^{0}$ the set of triangles $K$ in $\mathcal{T}_{h}$ which have no edges contained in $\partial \Omega$ and by $n_{h K}^{0}$ the number of such triangles. 
Lemma 7.1. In the case of dimension $d=2$, if $\Omega$ is simply-connected and if Hypothesis 4.1 holds, the dimension of the space $V_{h}^{T}$ is equal to $n_{h K}^{0}$, and it is spanned by the functions

$$
\boldsymbol{w}_{K}=\sum_{e \subset \partial K} \tilde{\mu}_{e} \boldsymbol{\tau}_{K e}, \quad K \in \mathcal{T}_{h}^{0}
$$

where the $\boldsymbol{\tau}_{K e}$ are the unit vectors orthogonal to $\boldsymbol{n}_{e}$ turning counterclockwise around $K$.

Proof. Let $n_{h K}, n_{h e}$ and $n_{h \boldsymbol{a}}$ denote the number of triangles in $\mathcal{T}_{h}$, the number of edges of these triangles and the number of their vertices, respectively. Similarly, let $n_{h e}^{b}$ be the number of these edges which are contained in $\partial \Omega$. It is readily checked that

$$
\operatorname{dim} X_{h}^{T}=n_{h e}-n_{h e}^{b}, \quad \operatorname{dim} M_{h}^{T 0}=n_{h \boldsymbol{a}}-1
$$

Moreover, since $\Omega$ is simply-connected, Euler-Poincaré's formula reads

$$
n_{h K}-n_{h e}+n_{h \boldsymbol{a}}=1
$$

and it follows from the inf-sup condition (4.1) that

$$
\operatorname{dim} V_{h}^{T}=\operatorname{dim} X_{h}^{T}-\operatorname{dim} M_{h}^{T 0}=n_{h e}-n_{h e}^{b}-n_{h \boldsymbol{a}}+1=n_{h K}-n_{h e}^{b} .
$$

Of course, this is equal to $n_{h K}^{0}$, which proves the first part of the lemma. Next, we observe that the number of functions $\boldsymbol{w}_{K}, K \in \mathcal{T}_{h}^{0}$, is $n_{h K}^{0}$ and that they are linearly independent, so it suffices to check that they belong to $V_{h}^{T}$.

We fix a $K$ in $\mathcal{T}_{h}^{0}$ and we observe that the support $\Omega_{K}$ of $\boldsymbol{w}_{K}$ is made of four triangles. The support of at most $\operatorname{six} \varphi_{a}$ intersect the interior of $\Omega_{K}$, and two situations occur:

1) If $\boldsymbol{a}$ is not a vertex of $K$, there exists a triangle $K^{\prime}$ which has a common edge $e$ with $K$ and contains $\boldsymbol{a}$. Then, it is readily checked that $\operatorname{grad} \varphi_{\boldsymbol{a}}$ is orthogonal to $\boldsymbol{\tau}_{K e}$, so that $b\left(\boldsymbol{w}_{K}, \varphi_{\boldsymbol{a}}\right)$ is zero.

2) If $\boldsymbol{a}$ is a vertex of $K$, it is the intersection of two edges $e$ and $e^{\prime}$, and, if $e^{\prime \prime}$ denotes the third edge of $K$, $\operatorname{grad} \varphi_{\boldsymbol{a}}$ is orthogonal to $\boldsymbol{\tau}_{K e^{\prime \prime}}$. So, we have to compute

$$
b_{h}\left(\boldsymbol{w}_{K}, \varphi_{\boldsymbol{a}}\right)=\int_{\Omega}\left(\tilde{\mu}_{e} \boldsymbol{\tau}_{K e}+\tilde{\mu}_{e^{\prime}} \boldsymbol{\tau}_{K e^{\prime}}\right) \cdot \operatorname{grad} \varphi_{\boldsymbol{a}} \mathrm{d} \boldsymbol{x} .
$$

Note that $\boldsymbol{\tau}_{K e} \cdot \operatorname{grad} \varphi_{\boldsymbol{a}}$ is constant on each triangle and continuous through $e$. The same property also holds for $e^{\prime}$. If $\Omega_{e}$ and $\Omega_{e^{\prime}}$ stand for the support of $\tilde{\mu}_{e}$ and $\tilde{\mu}_{e^{\prime}}$ respectively, this yields

$$
b_{h}\left(\boldsymbol{w}_{K}, \varphi_{\boldsymbol{a}}\right)=\left(\boldsymbol{\tau}_{K e} \cdot \operatorname{grad} \varphi_{\boldsymbol{a}}\right)_{\mid \Omega_{e}} \int_{\Omega} \tilde{\mu}_{e} \mathrm{~d} \boldsymbol{x}+\left(\boldsymbol{\tau}_{K e^{\prime}} \cdot \operatorname{grad} \varphi_{\boldsymbol{a}}\right)_{\mid \Omega_{e^{\prime}}} \int_{\Omega} \tilde{\mu}_{e^{\prime}} \mathrm{d} \boldsymbol{x} .
$$

Using (7.6), we obtain

$$
\begin{aligned}
b_{h}\left(\boldsymbol{w}_{K}, \varphi_{\boldsymbol{a}}\right) & =\left(\boldsymbol{\tau}_{K e} \cdot \operatorname{grad} \varphi_{\boldsymbol{a}}\right)_{\mid \Omega_{e}} \operatorname{meas}(e)+\left(\boldsymbol{\tau}_{K e^{\prime}} \cdot \operatorname{grad} \varphi_{\boldsymbol{a}}\right)_{\mid \Omega_{e^{\prime}}} \text { meas }\left(e^{\prime}\right) \\
& =\int_{e}\left(\boldsymbol{\tau}_{K e} \cdot \operatorname{grad} \varphi_{\boldsymbol{a}}\right) \mathrm{d} \tau+\int_{e^{\prime}}\left(\boldsymbol{\tau}_{K e^{\prime}} \cdot \operatorname{grad} \varphi_{\boldsymbol{a}}\right) \mathrm{d} \tau .
\end{aligned}
$$

This last quantity is equal to the integral on $\partial K$ of the tangential derivative of $\varphi_{\boldsymbol{a}}$, hence to zero. So, we obtain

$$
b_{h}\left(\boldsymbol{w}_{K}, \varphi_{\boldsymbol{a}}\right)=0,
$$

which ends the proof. 
Identifying the remaining part of $V_{h}$ is more complicated and requires a preliminary lemma. We now define, for all $e$ in $\mathcal{E}_{h}$ the function $\check{\mu}_{e}$ by

$$
\check{\mu}_{e}=\frac{1}{\operatorname{meas}(e)} \mu_{e} .
$$

We denote by $\mathcal{V}_{h}^{0}$ the set of vertices of triangles $K$ in $\mathcal{T}_{h}$ which do not belong to $\partial \Omega$ and by $n_{h \boldsymbol{a}}^{0}$ the number of such vertices.

Lemma 7.2. In the case of dimension $d=2$, if $\Omega$ is simply-connected, the dimension of the space of functions in $X_{h}^{N}$ which satisfy

$$
\forall q_{h} \in M_{h}^{N}, \quad b_{h}\left(\boldsymbol{v}_{h}, q_{h}\right)=0,
$$

is equal to $n_{h \boldsymbol{a}}^{0}$, and it is spanned by the functions

$$
z_{a}=\sum_{a \in e} \check{\mu}_{e} \boldsymbol{n}_{a e}, \quad \boldsymbol{a} \in \mathcal{V}_{h}^{0},
$$

where the $\boldsymbol{n}_{\boldsymbol{a}}$ are the unit vectors parallel to $\boldsymbol{n}_{e}$ turning counterclockwise around $\boldsymbol{a}$.

Proof. With the same notation as in the previous proof, it is readily checked that

$$
\operatorname{dim} X_{h}^{N}=n_{h e}-n_{h e}^{b}, \quad \operatorname{dim} M_{h}^{N 0}=n_{h K}-1 .
$$

Thanks to Euler-Poincaré's formula (7.8) and the inf-sup condition (5.6), the dimension of the space of functions in $X_{h}^{N}$ satisfying (7.10) is equal to $n_{h \boldsymbol{a}}-n_{h e}^{b}$, hence, since there are as many vertices contained in $\partial \Omega$ as edges, to $n_{h \boldsymbol{a}}^{0}$. Since the $n_{h \boldsymbol{a}}^{0}$ functions $\boldsymbol{z}_{\boldsymbol{a}}$ in (7.11) are linearly independent, it remains to check that they satisfy (7.10) or equivalently that

$$
\forall K \in \mathcal{T}_{h}, \quad b_{h}\left(\boldsymbol{z}_{\boldsymbol{a}}, \psi_{K}\right)=0 .
$$

Note that the support of $\boldsymbol{z}_{\boldsymbol{a}}$ is the union $\bar{\Omega}_{\boldsymbol{a}}$ of all triangles $K$ containing $\boldsymbol{a}$. So, let $K$ be a triangle contained in $\bar{\Omega}_{\boldsymbol{a}}$. Thanks to (3.10), we obtain by integration by parts

$$
b_{h}\left(\boldsymbol{z}_{\boldsymbol{a}}, \psi_{K}\right)=-\left(\operatorname{div} \boldsymbol{z}_{\boldsymbol{a}}\right)_{\mid K} \int_{K} \psi_{K} \mathrm{~d} \boldsymbol{x}=-\frac{1}{60} \int_{K} \operatorname{div} \boldsymbol{z}_{\boldsymbol{a}} \mathrm{d} \boldsymbol{x}=-\frac{1}{60} \sum_{e \subset \partial K} \int_{e} \boldsymbol{z}_{\boldsymbol{a}} \cdot \boldsymbol{n}_{e} \mathrm{~d} \tau,
$$

where for a while $\boldsymbol{n}_{e}$ is assumed to be exterior to $K$. If $e$ and $e^{\prime}$ denotes the two edges of $K$ which contain $\boldsymbol{a}$, this gives

$$
b_{h}\left(\boldsymbol{z}_{\boldsymbol{a}}, \psi_{K}\right)=-\frac{1}{60}\left(\int_{e} \check{\mu}_{e} \boldsymbol{n}_{\boldsymbol{a} e} \cdot \boldsymbol{n}_{e} \mathrm{~d} \tau+\int_{e^{\prime}} \check{\mu}_{e^{\prime}} \boldsymbol{n}_{\boldsymbol{a} e^{\prime}} \cdot \boldsymbol{n}_{e^{\prime}} \mathrm{d} \tau\right) .
$$

From the choice (7.9) of $\check{\mu}_{e}$, it is readily checked that $\int_{e} \check{\mu}_{e} \mathrm{~d} \tau=\int_{e^{\prime}} \check{\mu}_{e^{\prime}} \mathrm{d} \tau=1$, while one of the $\boldsymbol{n}_{\boldsymbol{a} e} \cdot \boldsymbol{n}_{e}$ and $\boldsymbol{n}_{\boldsymbol{a} e^{\prime}} \cdot \boldsymbol{n}_{e^{\prime}}$ is equal to 1 and the other to -1 . So, $b_{h}\left(\boldsymbol{z}_{\boldsymbol{a}}, \psi_{K}\right)$ vanishes, which concludes the proof.

We are now in a position to prove the main result of this section.

Theorem 7.3. In the case of dimension $d=2$, if $\Omega$ is simply-connected and if Hypothesis 4.1 holds, the dimension of the space $V_{h}$ is equal to $n_{h K}^{0}+n_{h a}^{0}$, and it is spanned by the functions $\boldsymbol{w}_{K}, K \in \mathcal{T}_{h}^{0}$, defined in (7.7), and by the functions

$$
\boldsymbol{w}_{\boldsymbol{a}}=\boldsymbol{z}_{\boldsymbol{a}}+\sum_{\boldsymbol{a} \in e} b_{h}\left(\boldsymbol{z}_{\boldsymbol{a}}, \varphi_{\boldsymbol{a}_{e}}\right) \tilde{\mu}_{e} \boldsymbol{\tau}_{\boldsymbol{a} e}, \quad \boldsymbol{a} \in \mathcal{V}_{h}^{0}
$$

where the $\boldsymbol{z}_{\boldsymbol{a}}$ are defined in (7.11), the $\boldsymbol{a}_{\boldsymbol{e}}$ denote the opposite endpoints to $\boldsymbol{a}$ on $e$ and the $\boldsymbol{\tau}_{\boldsymbol{a}}$ are the unit vectors orthogonal to $\boldsymbol{n}_{e}$ pointing towards $\boldsymbol{a}$. 
Proof. Since the dimension of $X_{h}$ is equal to $\operatorname{dim} X_{h}^{T}+\operatorname{dim} X_{h}^{N}$ and the dimension of $M_{h}^{0}$ is equal to $\operatorname{dim} M_{h}^{T 0}+$ $\operatorname{dim} M_{h}^{N 0}$ and since $M_{h}^{0}$ does not contain any spurious mode, evaluating the dimension of $V_{h}$ is clearly a consequence of Lemmas 7.1 and 7.2. Moreover the functions $\boldsymbol{w}_{K}$ belong to $V_{h}$ and, since the functions $\boldsymbol{z}_{\boldsymbol{a}}$ satisfy (7.10), the idea is to find functions $\boldsymbol{t}_{\boldsymbol{a}}$ in $X_{h}^{T}$ such that the functions $\boldsymbol{w}_{\boldsymbol{a}}=\boldsymbol{z}_{\boldsymbol{a}}+\boldsymbol{t}_{\boldsymbol{a}}$ satisfy

$$
\forall q_{h} \in M_{h}^{T}, \quad b_{h}\left(\boldsymbol{w}_{\boldsymbol{a}}, q_{h}\right)=0
$$

Indeed, the functions $\boldsymbol{w}_{K}, K \in \mathcal{T}_{h}^{0}$, and $\boldsymbol{w}_{\boldsymbol{a}}, \boldsymbol{a} \in \mathcal{V}_{h}^{0}$, form a linearly independent system. So, for a fixed $\boldsymbol{a}$, since the support of $\boldsymbol{z}_{\boldsymbol{a}}$ is $\bar{\Omega}_{\boldsymbol{a}}$, we look for a function $\boldsymbol{t}_{\boldsymbol{a}}$ of the form

$$
\boldsymbol{t}_{\boldsymbol{a}}=\sum_{\boldsymbol{a} \in e} \alpha_{e} \tilde{\mu}_{e} \boldsymbol{\tau}_{\boldsymbol{a} e}
$$

Next, for all edges $e^{\prime}$ containing $\boldsymbol{a}$, it is readily checked that

$$
b_{h}\left(\boldsymbol{t}_{\boldsymbol{a}}, \varphi_{\boldsymbol{a}_{e^{\prime}}}\right)=\alpha_{e^{\prime}}\left(\operatorname{grad} \varphi_{\boldsymbol{a}_{e^{\prime}}} \cdot \boldsymbol{\tau}_{\boldsymbol{a} e^{\prime}}\right)_{\mid e^{\prime}} \int_{\Omega_{a}} \tilde{\mu}_{e^{\prime}} \mathrm{d} \boldsymbol{x} .
$$

Using (7.6) and the fact that $\left(\operatorname{grad} \varphi_{\boldsymbol{a}_{e^{\prime}}} \cdot \boldsymbol{\tau}_{\boldsymbol{a} e^{\prime}}\right)_{\mid e^{\prime}}$ is equal to - meas $\left(e^{\prime}\right)^{-1}$, we obtain

$$
b_{h}\left(\boldsymbol{t}_{\boldsymbol{a}}, \varphi_{\boldsymbol{a}_{e^{\prime}}}\right)=-\alpha_{e^{\prime}}
$$

So the choice (7.12) implies that all the $b_{h}\left(\boldsymbol{w}_{\boldsymbol{a}}, \varphi_{\boldsymbol{a}_{e^{\prime}}}\right)$ are equal to zero, and finally, since $\operatorname{grad} \varphi_{\boldsymbol{a}}$ is equal to $-\sum_{\boldsymbol{a} \in e} \operatorname{grad} \varphi_{\boldsymbol{a}_{e}}$ on $\Omega_{\boldsymbol{a}}$, that $b_{h}\left(\boldsymbol{w}_{\boldsymbol{a}}, \varphi_{\boldsymbol{a}}\right)$ also vanishes. This concludes the proof.

Note that both functions $\boldsymbol{w}_{K}$ and $\boldsymbol{w}_{\boldsymbol{a}}$ have local supports. More precisely,

- the support of each $\boldsymbol{w}_{K}$ is made of four triangles,

- the support of each $\boldsymbol{w}_{\boldsymbol{a}}$ is made of a finite number of triangles, and the maximal number only depends on the regularity parameter $\sigma$.

In both cases, the diameter of this support is bounded by a constant times the diameter of any triangle $K$ contained in it. Moreover a basis of $W_{h}$, made of functions with local supports is easy to construct from the definition of $\boldsymbol{w}_{K}$ and $\boldsymbol{w}_{\boldsymbol{a}}$.

The arguments for extending the characterization of $V_{h}$ to the case of dimension $d=3$ can be found in [12, Chap. 5]. However they lead to very technical proofs for the present element, so that we have rather skip the three-dimensional results.

Acknowledgements. The authors wish to thank the Département de Thermohydraulique et de Physique of the Commissariat à l'Énergie Atomique in Grenoble for suggesting them the study of this new element, developed in the project PRICELES. They also thank Professors M. Crouzeix and P.-A. Raviart for letting them add so much pressure to their element.

\section{REFERENCES}

[1] K. Arrow, L. Hurwicz and H. Uzawa, Studies in Nonlinear Programming. Stanford University Press, Stanford (1958).

[2] I. Babuška, The finite element method with Lagrangian multipliers. Numer. Math. 20 (1973) 179-192.

[3] C. Bergé, Théorie des graphes. Dunod, Paris (1970).

[4] J. Boland and R. Nicolaides, Stability of finite elements under divergence constraints. SIAM J. Numer. Anal. 20 (1983) $722-731$.

[5] F. Brezzi, On the existence, uniqueness and approximation of saddle-point problems arising from Lagrange multipliers. RAIRO - Anal. Numér. 8 R2 (1974) 129-151.

[6] P.G. Ciarlet, Basic Error Estimates for Elliptic Problems, in the Handbook of Numerical Analysis, Vol. II, P.G. Ciarlet and J.-L. Lions Eds., North-Holland, Amsterdam (1991) 17-351. 
[7] P. Clément, Développement et applications de méthodes numériques volumes finis pour la description d'écoulements océaniques. Thesis, Université Joseph Fourier, Grenoble (1996).

[8] M. Crouzeix and P.-A. Raviart, Conforming and nonconforming finite element methods for solving the stationary Stokes equations. RAIRO - Anal. Numér. 7 R3 (1973) 33-76.

[9] P. Emonot, Méthodes de volumes éléments finis: application aux équations de Navier-Stokes et résultats de convergence. Thesis, Université Claude Bernard, Lyon (1992).

[10] M. Fortin, An analysis of the convergence of mixed finite element methods. RAIRO - Anal. Numér. 11 R3 (1977) 341-354.

[11] V. Girault and P.-A. Raviart, Finite Element Methods for the Navier-Stokes Equations, Theory and Algorithms. SpringerVerlag, Berlin (1986).

[12] F. Hecht, Construction d'une base d'un élément fini $P_{1}$ non conforme à divergence nulle dans $\mathbb{R}^{3}$. Thesis, Université Pierre et Marie Curie, Paris (1980)

[13] F. Hecht, Construction d'une base de fonctions $P_{1}$ non conforme à divergence nulle dans $\mathbb{R}^{3}$. RAIRO - Anal. Numér. $\mathbf{1 5}$ (1981) 119-150.

[14] R. Verfürth, Error estimates for a mixed finite element approximation of the Stokes equations. RAIRO - Anal. Numér. 18 (1984) 175-182.

To access this journal online:

www.edpsciences.org 